ARTICLE

https://doi.org/10.1038/s41467-019-11606-x

\title{
Protein prenylation restrains innate immunity by inhibiting Rac1 effector interactions
}

\author{
Murali K. Akula (1) 1,2, Mohamed X. Ibrahim¹,10, Emil G. Ivarsson¹,10, Omar M. Khan (10 3,4,10, Israiel T. Kumar1, \\ Malin Erlandsson ${ }^{5}$, Christin Karlsson ${ }^{1}$, Xiufeng $\mathrm{Xu}^{6}$, Mikael Brisslert ${ }^{5}$, Cord Brakebusch7, Donghai Wang ${ }^{8}$, \\ Maria Bokarewa (1) ${ }^{5}$, Volkan I. Sayin ${ }^{2,9}$ \& Martin O. Bergo ${ }^{1,6}$
}

Rho family proteins are prenylated by geranylgeranyltransferase type I (GGTase-I), which normally target proteins to membranes for GTP-loading. However, conditional deletion of GGTase-I in mouse macrophages increases GTP-loading of Rho proteins, leading to enhanced inflammatory responses and severe rheumatoid arthritis. Here we show that heterozygous deletion of the Rho family gene Rac1, but not Rhoa and Cdc42, reverses inflammation and arthritis in GGTase-I-deficient mice. Non-prenylated Rac1 has a high affinity for the adaptor protein Ras GTPase-activating-like protein 1 (Iqgap1), which facilitates both GTP exchange and ubiquitination-mediated degradation of Rac1. Consistently, inactivating lagap1 normalizes Rac1 GTP-loading, and reduces inflammation and arthritis in GGTase-I-deficient mice, as well as prevents statins from increasing Rac1 GTP-loading and cytokine production in macrophages. We conclude that blocking prenylation stimulates Rac1 effector interactions and unleashes proinflammatory signaling. Our results thus suggest that prenylation normally restrains innate immune responses by preventing Rac1 effector interactions.

\footnotetext{
${ }^{1}$ Sahlgrenska Cancer Center, Department of Molecular and Clinical Medicine, Institute of Medicine, University of Gothenburg, SE-405 30 Gothenburg, Sweden. ${ }^{2}$ Wallenberg Centre for Molecular and Translational Medicine, University of Gothenburg, SE-405 30 Gothenburg, Sweden. ${ }^{3}$ Adult Stem Cell Laboratory, Francis Crick Research Institute, London NW1 1AT, UK. ${ }^{4}$ College of Health and Life Sciences, Hamad Bin Khalifa University, Education City, Qatar Foundation, Doha 34110, Qatar. ${ }^{5}$ Department of Rheumatology, Institute of Medicine, University of Gothenburg, SE-41345 Gothenburg, Sweden. ${ }^{6}$ Department of Biosciences and Nutrition, Karolinska Institutet, SE-141 83 Huddinge, Sweden. ${ }^{7}$ Biotech Research and Innovation Centre, University of Copenhagen, 2200 Copenhagen N, Denmark. ${ }^{8}$ Department of Immunology, Duke University School of Medicine, Durham, NC 27710, USA. ${ }^{9}$ Sahlgrenska Cancer Center, Department of Surgery, Institute of Clinical Sciences, University of Gothenburg, SE-405 30 Gothenburg, Sweden. ${ }^{10}$ These authors contributed equally: Mohamed X. Ibrahim, Emil G. Ivarsson, Omar M. Khan. Correspondence and requests for materials should be addressed to M.O.B. (email: martin.bergo@ki.se)
} 
P rotein geranylgeranyltransferase type I (GGTase-I) transfers a 20-carbon geranylgeranyl lipid to a cysteine residue of proteins harboring a carboxyl-terminal CAAX motif, including the Rho family proteins Rac1, RhoA, and Cdc42 ${ }^{1}$. Geranylgeranylation, also called prenylation, enhances hydrophobicity and facilitates membrane anchoring of Rho proteins and is believed to be essential for correct subcellular targeting, effector binding, GTP loading, and activation ${ }^{2,3}$.

Geranylgeranylation is an evolutionarily conserved modification that has generated a broad interest for several reasons. First, Rho family proteins contribute to tumor growth and metastasis, which prompted the development of GGTase-I inhibitors (GGTIs) ${ }^{4}$. Several GGTIs exhibit anti-tumor effects in preclinical studies and the rationale for using GGTIs in cancer therapy is supported by mouse gene-targeting experiments ${ }^{5,6}$. Second, Rho proteins regulate phagocytosis, migration, reactive oxygen species (ROS) production, and signaling in inflammatory cells ${ }^{7}$. Thus, targeting GGTase-I has been proposed as a strategy to treat inflammatory and autoimmune disorders, including rheumatoid arthritis and multiple sclerosis ${ }^{8-10}$. And third, reduced geranylgeranylation of Rho proteins is frequently suggested to underlie anti-inflammatory properties and other pleiotropic effects of statins ${ }^{11,12}$. Statins lower cholesterol levels by blocking mevalonate synthesis but this also leads to reduced synthesis of geranylgeranylpyrophosphate (GGPP), the lipid substrate of GGTase-I ${ }^{13}$.

The idea that blocking Rho protein geranylgeranylation would inhibit inflammation was challenged by studies into GGTase-Ideficient mice ${ }^{14,15}$. Knockout of GGTase-I's catalytic subunit in macrophages eliminated Rho protein geranylgeranylation, but surprisingly, Rac1, RhoA, and Cdc42 accumulated in their GTPbound active form, and Racl remained associated with membranes $^{14}$. Moreover, p38 and $\mathrm{NFKB}$ activities were high in GGTase-I-deficient macrophages, which increased proinflammatory cytokine production after lipopolysaccharide (LPS) stimulation; and the mice developed chronic erosive rheumatoid arthritis ${ }^{14}$. Thus, targeting macrophage GGTase-I activates Rho proteins and causes inflammation.

These results dispute the general understanding of the biochemical and medical importance of $C A A X$ protein geranylgeranylation and raise a range of new questions ${ }^{16}$. For example, GGTase-I has more than 60 predicted substrates ${ }^{17}$, and it is not yet known whether one or more of these proteins mediate inflammatory signaling and erosive arthritis development in mice lacking GGTase-I in macrophages. It would also be important to define mechanisms underlying the increased GTP loading of nonprenylated Rho proteins. One potential explanation is that they interact more avidly than prenylated Rho proteins with a guanine-nucleotide exchange factor (GEF) or an adaptor protein such as Ras GTPase-activating-like protein (Iqgap1) ${ }^{18-20}$. Alternatively, nonprenylated Rho proteins interact less avidly with a GTPase-activating protein $(\mathrm{GAP})^{18}$ or guanine nucleotide dissociation inhibitor (RhoGDI1) ${ }^{21}$. Interestingly, knockdown of Rhogdi1 increases GTP loading of Rho family proteins ${ }^{22}$, and it is possible that a reduced interaction with RhoGDI1 underlies Rhoprotein activation and inflammation in GGTase-I-deficient macrophages. Moreover, it would be important to establish whether statins, by reducing GGPP levels, produce similar effects in macrophages as the knockout of GGTase-I. Statin administration is frequently associated with increased Rho-GTP loading and sometimes with increased LPS-induced cytokine production, including interleukin (Il) $1 \beta$, but the underlying mechanism is unknown $^{23}$. In the current study, we used genetic, pharmacologic, and proteomic strategies to address those issues and identify a new potential explanation for the role of prenylation for Rac1 effector interactions and proinflammatory signaling.

\section{Results}

Rac1 knockout prevents arthritis in GGTase-I-deficient mice. Mice lacking GGTase-I in macrophages (Pggt1 $b^{\mathrm{fl} / \mathrm{fl}}$ LysM-Cre ${ }^{+/ 0}$, hereafter designated $\operatorname{Pggt}_{1} b^{\Delta / \Delta}$ ) develop erosive arthritis, and nonprenylated Rac1, RhoA, and Cdc42 accumulate in the GTPbound state ${ }^{14}$. In TX-114 phase-separation assays Rac1, RhoA, and Cdc42 exhibited a substantial shift from the detergent to aqueous phase in $P g g t 1 b^{\Delta / \Delta}$ macrophages; and click-chemistry experiments revealed that Racl is not geranylgeranylated-thus confirming the previous conclusion that these proteins are not prenylated (Supplementary Fig. 1A and B) ${ }^{14}$. We hypothesized that the increased activity of one of these nonprenylated proteins mediates arthritis development and the proinflammatory phenotypes of $\operatorname{Pggt}_{1} b^{\Delta / \Delta}$ mice. To test this hypothesis, we knocked out one copy of Rac1, Rhoa, and Cdc42 in macrophages of $\operatorname{Pggt}_{1 b^{\Delta / \Delta}}$ mice (previous studies show that knockout of both copies of Rac1, Rhoa, and Cdc42 produces multiple in vivo and cellular phenotypes) ${ }^{24-29}$. As expected, levels of total Rac1, RhoA, and Cdc42 were $~ 50 \%$ (47-58\%) lower in BM macrophages from

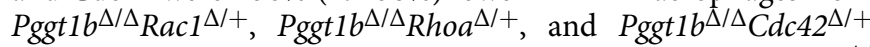
mice, respectively, than in macrophages from littermate Pggt $1 b^{\Delta /}$ $\Delta$ mice (Supplementary Fig. 1C). Similarly, levels of GTP-bound Rac1 was $\sim 50 \%$ lower in $\operatorname{Pggt}_{1} b^{\Delta / \Delta} \mathrm{Rac1}^{\Delta /+}$ than in $\operatorname{Pggt}^{\mathrm{d}} b^{\Delta / \Delta}$ macrophages (Fig. 1a). Immunohistochemical analyses revealed that the high synovitis and bone erosion scores in joints of $\operatorname{Pggt}_{1} b^{\Delta / \Delta}$ mice were markedly lower in $\operatorname{Pggt} 1 b^{\Delta / \Delta} R a c 1^{\Delta /+}$ mice, and were statistically indistinguishable from wild type (Fig. 1b). The arthritis scores were similar in $\operatorname{Pggt}_{1} b^{\Delta / \Delta}$ Rhoa $^{\Delta /+}$ and $\operatorname{Pggt}_{1} b^{\Delta / \Delta}$ mice whereas they were higher in $\operatorname{Pggt}_{1 b^{\Delta / \Delta}} C d c 42^{\Delta /+}$ mice (Supplementary Fig. 1D and E).

As in earlier studies, LPS-stimulated $P g g t 1 b^{\Delta / \Delta}$ macrophages produced and secreted Il-1 $\beta$ (which wild-type macrophages do not); this effect was associated with caspase-1 activation (Fig. 1c, d). Inactivation of Rac1 reduced caspase- 1 and Il-1 $\beta$ production by $90-100 \%$ (Fig. 1c, d). LPS-stimulated $P g g t 1 b^{\Delta / \Delta}$ macrophages also secreted high amounts of 1l-6, Tnf, and Mmp13; whereas the levels in medium of $\operatorname{Pggt} b^{\Delta / \Delta} \mathrm{RaCl}^{\Delta /+}$ and wild-type macrophages were similar (Fig. 1c, e). In addition, basal expression of inflammation and extracellular matrix-associated genes were increased in $P g g t 1 b^{\Delta / \Delta}$ macrophages, but similar to wild-type in Pggt1b ${ }^{\Delta / \Delta}$ Rac1 $1^{\Delta /+}$ cells (Supplementary Fig. $1 \mathrm{~F}$ and G). Cytokine

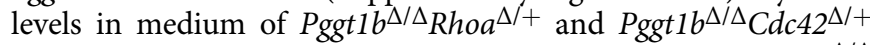
macrophages did not differ consistently compared with Pggt $1 b^{\Delta / \Delta}$ (Supplementary Fig. 2A-D).

Pggt $1 b^{\Delta / \Delta}$ macrophages exhibited increased levels of LPSstimulated p38 phosphorylation and phosphorylation of the Nf$\kappa \mathrm{B}$-regulator I $\mathrm{BB}$ kinase (Ikk); and increased basal and LPSstimulated phosphorylation of the tyrosine protein kinase Src and signal transducer and activator of transcription 3 (Stat3) (Fig. 1f). Consistent with results in Fig. 1a-e, phosphorylation of p38, Src, Ikk, and Stat3 was reduced in $\operatorname{Pggt} 1 b^{\Delta / \Delta} \operatorname{Racl}^{\Delta /+}$ macrophages (Fig. 1f).

Rac1-GTP may activate inflammatory signaling pathways through multiple effectors including ROS and p38. Pharmacological inhibition of ROS-reduced phosphorylation of Stat3, Src, and Ikk in $P g g t 1 b^{\Delta / \Delta}$ macrophages, but did not influence p38 phosphorylation (Supplementary Fig. 2E and F). Moreover, a p38 inhibitor and a ROS inhibitor prevented the increased cytokine production observed in LPS-stimulated $\operatorname{Pggt}_{1} b^{\Delta / \Delta}$ macrophages (Fig. $1 \mathrm{~g}$ and Supplementary Fig. $2 \mathrm{G}$ and $\mathrm{H}$ ).

GGTase-I knockout increases Rac1-GTP, but reduces total Rac1. Nonprenylated Rac1, RhoA, and Cdc42 in Pggt $1 b^{\Delta / \Delta}$ macrophages accumulate in their GTP-bound active forms ${ }^{14}$ (Fig. 2a and Supplementary Fig. 3A and B); gene-expression levels 
a

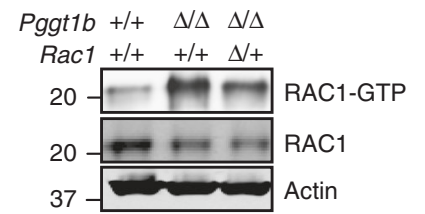

C

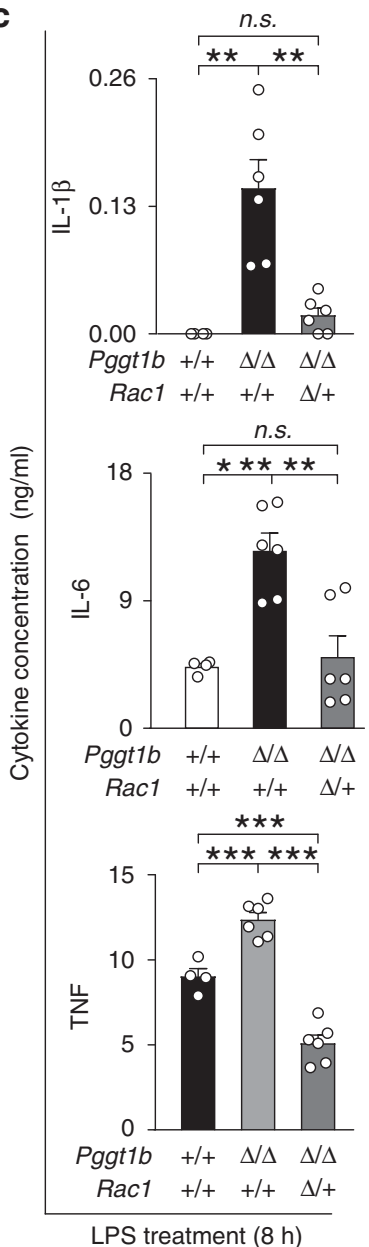

b
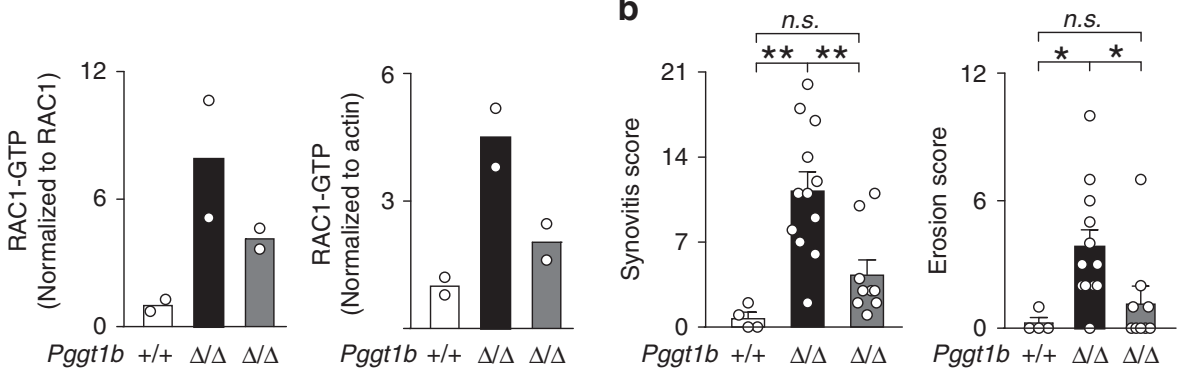

Rac1 $+/++/+\Delta /+$

Rac1 $+/+\quad+/+\Delta /+$

Rac1 $+/++/+\Delta /+$

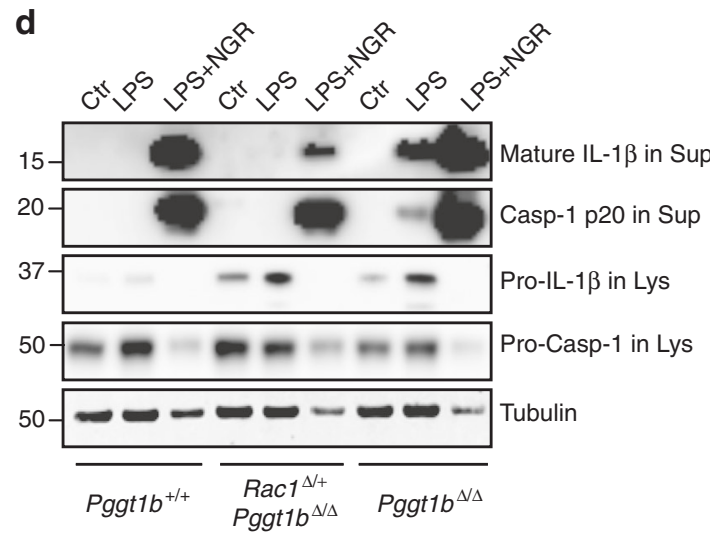

f

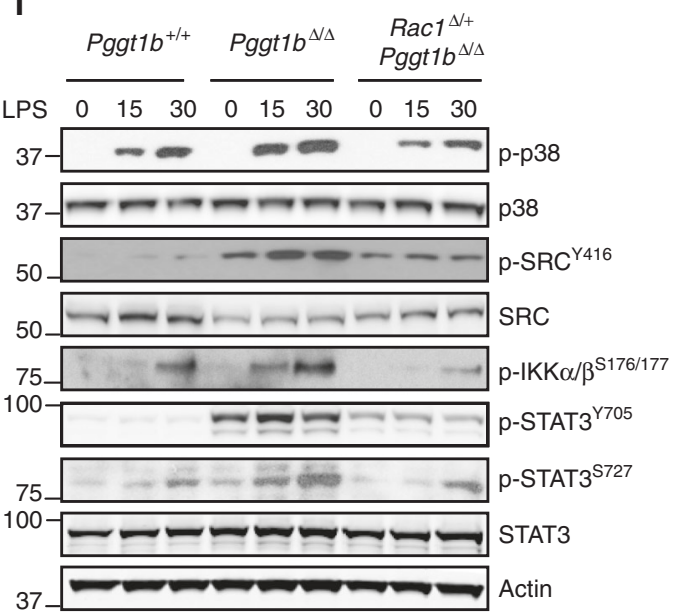

e g

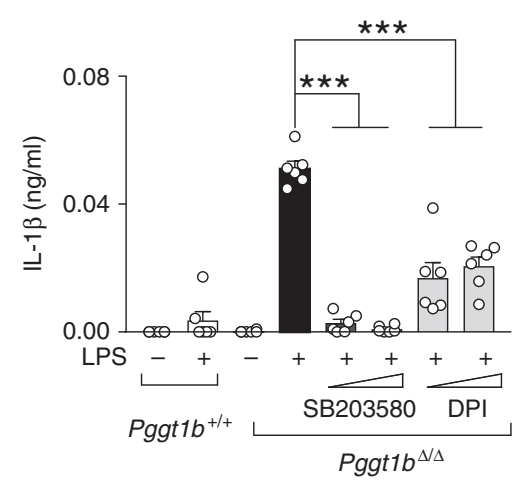

Fig. 1 Rac1 haploinsufficiency rescues arthritis and inflammatory signaling in Pggtib ${ }^{\Delta / \Delta}$ mice. a Left, Western blots showing steady-state levels of GTPbound and total Rac1 in BM macrophages isolated from Pggt $1 b^{\Delta /+}$, Pggt $1 b^{\Delta / \Delta}$, and littermate Rac ${ }^{\Delta /+}$ Pggt $7 b^{\Delta / \Delta}$ mice. Actin was used as a loading control. Right, Bar graphs showing mean Rac1-GTP levels determined by densitometry ( $n=2$ per genotype). $\mathbf{b}$ Synovitis and erosion score in joints of 12 -week-old $\mathrm{Pggt}_{1} \mathrm{~b}^{+/+}(n=4), \operatorname{Pggt7}^{\Delta / \Delta}(n=12)$, and $\operatorname{Rac}^{\Delta /+} \operatorname{Pggt7b}^{\Delta / \Delta}(n=9)$ mice. c Cytokine concentrations, $8 \mathrm{~h}$ after LPS (10 ng/ml) stimulation, in medium of primary bone marrow (BM) macrophages isolated from Pggt $1 b^{+/+}(n=3)$, Pggt $1 b^{\Delta / \Delta}(n=4)$, and Rac $7^{\Delta /+} \operatorname{Pggt}^{1} b^{\Delta / \Delta}(n=3)$ mice. d Western blots showing levels of mature II-1 $\beta$ and caspase- 1 in supernatants (Sup), and pro-II-1 $\beta$ and pro-caspase-1 in lysates (Lys) of LPS (200 ng/ml) stimulated BM macrophages; tubulin in lysates was used as a loading control. The antibiotic nigericin ( $28 \mathrm{mM}$ ) was used as a positive control for inflammasome-mediated caspase-1 activation and II-1 $\beta$ production. e Western blot showing levels of Mmp13 in medium of LPS-stimulated BM macrophages; Actin in lysates was used as a loading control. $\mathbf{f}$ Western blots showing phosphorylated $(p)$ and total levels of intracellular signaling mediators in lysates of BM macrophages isolated 0,15 , and 30 min after LPS stimulation. $\mathbf{g}$ Concentration of II- $1 \beta$ in medium of LPS-stimulated BM macrophages ( $n=3 /$ genotype) that had been pre-incubated for $1 \mathrm{~h}$ with inhibitors of p38 (SB203580; 1 and $5 \mu \mathrm{M}$ ) and ROS (DPI; $500 \mathrm{nM}$ and $5 \mu \mathrm{M}$ ). For c-e, g, similar results were observed in two to three independent experiments. Error bars presented as s.e.m. when $n$ is equal to or more than three. Significance between groups were calculated with two tailed Student's $t$ test $(\mathbf{c}, \mathbf{g})$ and one-way ANOVA with Tukey's post hoc test (b). n.s. not significant, ${ }^{\star} P<0.05,{ }^{\star \star} P<0.01,{ }^{\star \star \star} P<0.001$

were unaffected (Fig. 2b). Further analyses revealed that the total levels of Rac1 were reduced by $\sim 50 \%$ (Fig. 2a). Moreover, the half-life of Racl was $65-70 \%$ shorter in Pggt $1 b^{\Delta / \Delta}$ than in control cells (Fig. 2c, d). To determine whether ubiquitin-mediated proteasomal degradation contributed to the reduced Rac1 levels, we first immunoprecipitated ubiquitin and performed western blots for Rac1; and found higher amounts of ubiquitin-associated Rac1 in $P g g t 1 b^{\Delta / \Delta}$ than in control lysates (Fig. 2e). Although we 
a
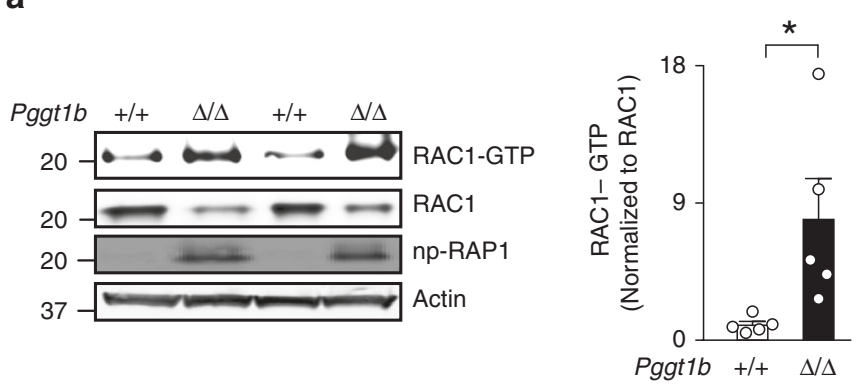

C

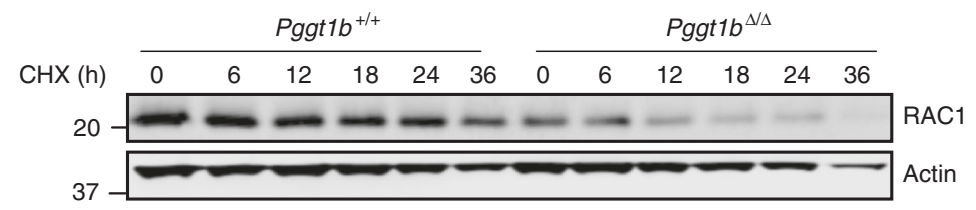

d
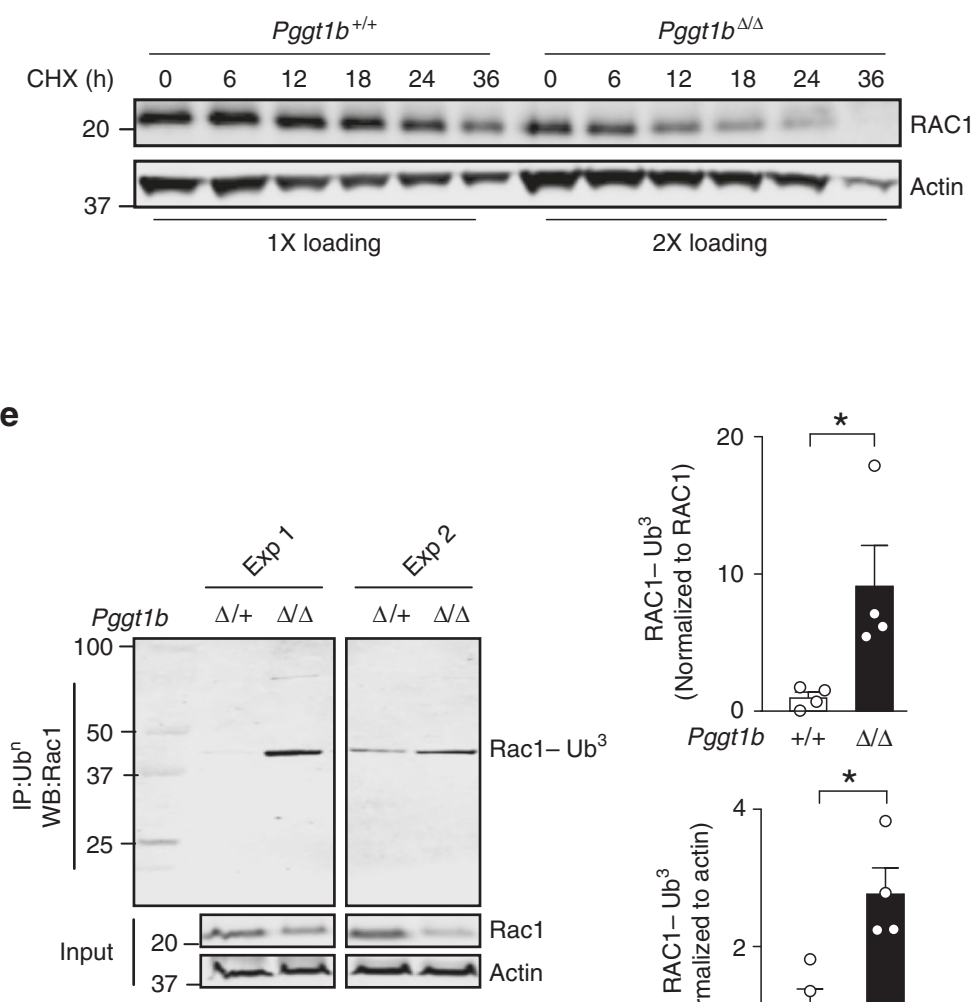

e

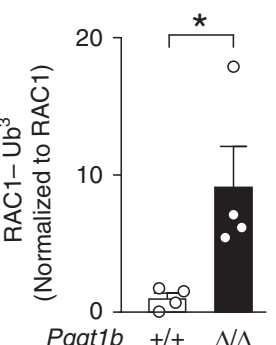

b

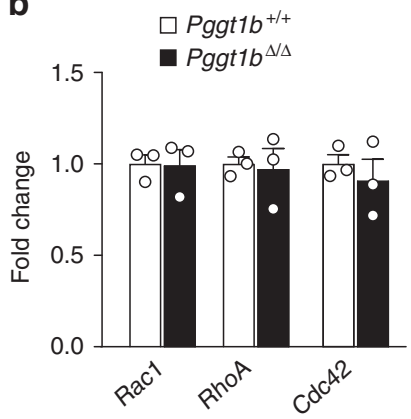

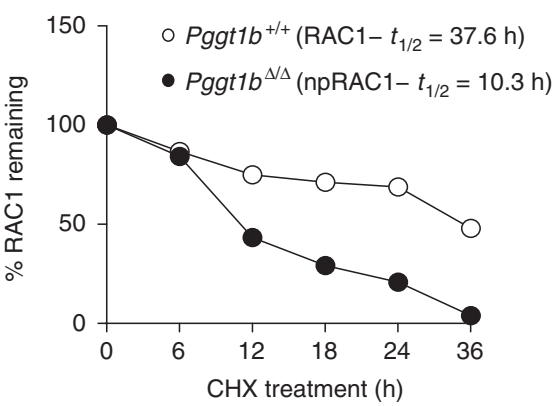

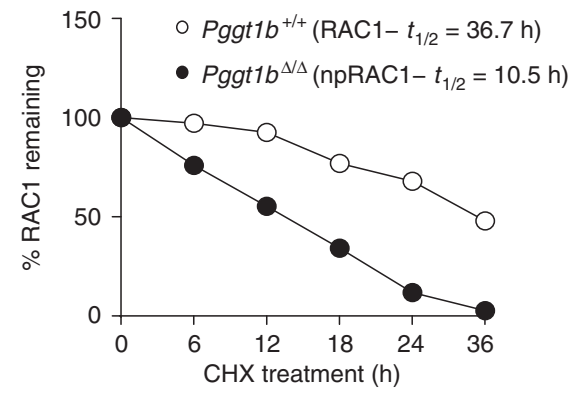

f
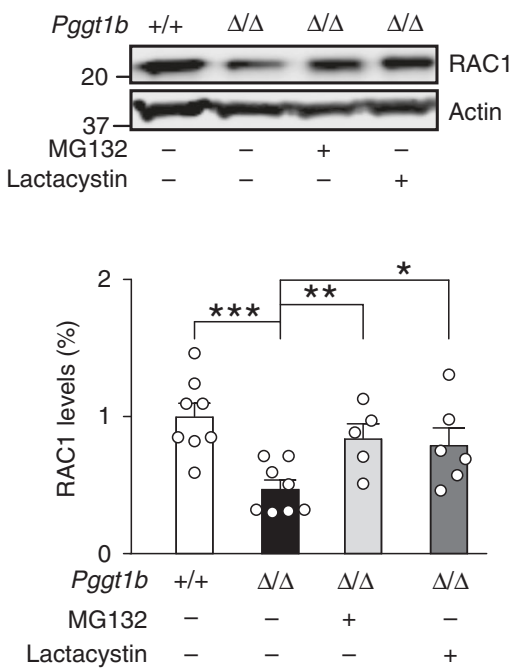

only detected Racl conjugated with three Ubiquitins under the current experimental conditions, we can't rule out the existence of mono-, di-, and polyubiquitinated forms. Second, we incubated macrophages with proteasome inhibitors and found that total Rac1 levels were restored (Fig. 2f); proteasome inhibition also increased Rac1-GTP levels (Supplementary Fig. 3C). In contrast with Rac1, total RhoA, and Cdc42 levels were increased in Pggt $1 b^{\Delta / \Delta}$ cells (Supplementary Fig. $3 \mathrm{~A}$ and B). Thus, blocking prenylation reduces Racl stability but appears to increase that of RhoA and Cdc42. 
Fig. 2 GGTase-I knockout increases Rac1-GTP loading, but reduces Rac1 total levels. a Left, western blots showing steady-state levels of GTP-bound and total Rac1 in BM macrophages. Nonprenylated RAP1A was used as marker of GGTase-I-deficient cells; actin was used as a loading control. Middle and right, amount of GTP-bound ( $n=5$ /genotype) and total Rac1 ( $n=17 /$ genotype) in BM macrophages determined by densitometry of protein bands. b Quantitative polymerase chain reaction (QPCR) data showing levels of Rac1, Rhoa, and Cdc42 expression in cDNA of BM macrophages ( $n=3$ per genotype). c Left, western blots showing levels of Rac1 and Actin that remain in BM macrophages at various time points after incubation with cycloheximide $(20 \mu \mathrm{g} / \mathrm{ml})$ to stop protein synthesis. Equal amounts of total proteins were loaded. Right, densitometry of protein bands normalized to timepoint 0 within each genotype. $\mathbf{d}$ Similar experiment as in (c) except twice the amount of total proteins from the Pggt $7 b^{\Delta / \Delta}$ lysates were loaded compared to $P_{g g t} 1 b^{\Delta /+}$ to obtain similar Rac1 levels at time-point 0. e Left, immunoprecipitation (IP) of Ubiquitin (Ub) followed by western blots for Rac1. Direct western blots were performed on the same lysates (input) to quantify total levels of Rac1 and Actin. The molecular weight of the main band was $\sim 45 \mathrm{kDa}$ which corresponds to Rac1 conjugated with three Ubs. Two independent experiments are shown. Right, amount of ubiquitin-bound Rac1 determined by densitometry of protein bands ( $n=4$ /genotype). $\mathbf{f}$ Upper panel, western blots showing total Rac1 levels in lysates of BM macrophages incubated for $10 \mathrm{~h}$ with proteasome inhibitors MG-132 $(15 \mu \mathrm{M})$ and lactacystin $(15 \mu \mathrm{M})$. Lower panel, quantification of protein bands normalized to Actin and expressed as percent of control $\left(\operatorname{Pggt}_{7} b^{\Delta /+}\right)$. Similar results were obtained three times. Error bars represent s.e.m. Significance between groups were calculated with two tailed Student's $t$ test. ${ }^{\star} P<0.05,{ }^{\star \star} P<0.01,{ }^{\star \star \star} P<0.001$

\begin{tabular}{|c|c|c|c|c|c|}
\hline S No. & Accession & Description & PSM & Fold change & $P$ value \\
\hline 2 & Q640N3 & Rho GTPase-activating protein 30 [RHG30] & 5 & 1.12 & 0.012 \\
\hline 3 & Q8BYW1 & Rho GTPase-activating protein 25 [RHG25] & 2 & 1.60 & 0.043 \\
\hline 4 & A6X8Z5 & Rho GTPase-activating protein 31 [RHG31] & 9 & 0.85 & 0.170 \\
\hline
\end{tabular}

RhoGDI1 is not involved in phenotypes of GGTase-I deficiency. The GGTase-I knockout reduces interactions between RhoGDI1 and Rac1 and Cdc42, but not RhoA ${ }^{14}$. We hypothesized that a reduced interaction between RhoGDI1 and Rac1 might underlie increased GTP loading and cytokine production and tested this by inactivating RhoGDI1 expression. Consistent with a previous study ${ }^{22}$, suppressing RhoGDI1 expression with small-interfering (si) RNAs increased Rac1 GTP loading and reduced total Racl levels-a result that was associated with increased LPS-stimulated Il-6 and Tnf production (Supplementary Fig. 4A). However, we only observed this result in the RAW264.7 macrophage cell line; it was neither observed in primary macrophages incubated with siRNAs, nor in immortalized macrophages where the Arhgdial gene had been inactivated with CRISPR/CAS9 (Supplementary Fig. $4 \mathrm{~B}$ and C). Moreover, RhoGDI1 inactivation never increased Il-1 $\beta$ production, including in RAW264.7 cells (Supplementary Fig. 4A-C).

Iqgap1 binds nonprenylated Racl and mediates arthritis. To determine whether blocking Rac1 prenylation influences other effector interactions, we immunoprecipitated Racl from Pggt $1 b^{\Delta /}$ $\Delta$ and control macrophage lysates and performed isobaric tagging for relative peptide quantification with mass spectrometry ${ }^{30}$. From a list of 717 proteins whose levels differed significantly in $\operatorname{Pggt}_{1} b^{\Delta / \Delta}$ and control cells, we identified five known Rho family effector proteins (Table 1). The top hit was GTPase-activatinglike protein 1 (Iqgap1) -an adaptor protein that binds and stabilizes GTP-bound Rho family proteins but lacks GAP and GEF activity ${ }^{20,31,32}$. Immunoprecipitation (IP) and western blot analyses of macrophage lysates revealed that the Rac1-Iqgap1 interaction was two to threefold higher in $P g g t 1 b^{\Delta / \Delta}$ than control cells (Fig. 3a). The interaction between Iqgap1 and RhoA and Cdc42 in Pggt $1 b^{\Delta / \Delta}$ macrophages was also increased (Supplementary Fig. 5A and B).

To determine whether Iqgap1 contributes to phenotypes of GGTase-I deficiency, we bred Pggt $1 b^{\Delta / \Delta}$ mice on an Iqgap $1^{-1-}$ background ${ }^{33}$. Levels of synovitis and bone erosion were $60-70 \%$ lower in joints of $P_{g g t} 1 b^{\Delta / \Delta}$ Iqgap $^{-/-}$than Pggt 16 ${ }^{\Delta / \Delta}$ Iqgap $1^{+/+}$mice, and cytokine production by LPSstimulated Pggt1 $b^{\Delta / \Delta}$ Iqgap $1^{-1-}$ macrophages was reduced to control levels (Fig. 3b, c). Importantly, knockout of Iqgap1 alone (e.g., Pggt1b ${ }^{+/+}$Iqgap $1^{-7-}$ ) did not influence cytokine production (Supplementary Fig. 5C), although it reduced basal Rac1-GTP and total Rac1 levels (Supplementary Fig. 5D). Moreover, the Iqgap1 knockout reduced Rac1-GTP loading and ubiquitination, and increased total Racl to levels observed in controls; and essentially normalized LPS-induced p38, Src, and Stat3 phosphorylation (Fig. 3d, e, and Supplementary Fig. 5E). The Iqgap1 knockout also reduced GTP-bound and total RhoA and Cdc42 to control levels (Supplementary Fig. 5F and G). Furthermore, Pggt $1 b^{\Delta / \Delta}$ macrophages have a small adhesive area and appear small and rounded, and the knockout of Iqgap1 abolished this phenotype (Fig. 3f).

Tiam1 binds nonprenylated Rac1 and stimulates GTP loading. Iqgap1 has no GEF or GAP activity ${ }^{20,31}$. Thus, we asked whether an increased interaction with a known GEF-or a reduced interaction with RacGAP1 - could explain the increased GTP loading of nonprenylated Rac1. IP-western blot analyses revealed a two to sixfold higher Rac1-Tiam1 interaction in Pggt1 $b^{\Delta / \Delta}$ than in control macrophages (Fig. 4a). Interactions between Rac1 and the GEFs Vav1, Vav2, $\beta$-Pix, and Dock1, and the GAP RacGAP1 were similar in Pggt 1b $b^{\Delta / \Delta}$ and control macrophages (Supplementary Fig. 6A). Inhibiting Tiam1 expression with siRNAs reduced GTP-bound and increased total Rac1 (Fig. 4b). Tiam1 inhibition also reduced cytokine production of LPS-stimulated Pggt $1 b^{\Delta / \Delta}$ macrophages (Fig. 4c and Supplementary Fig. 6B and C).

To determine whether Tiam1 interacts with Iqgap1, we performed IP-western blot analyses and found a low basal interaction in control Pggt $1 b^{+/+}$macrophages and a high degree of interaction in Pggt $1 b^{\Delta / \Delta}$ macrophages (Fig. 4d). These experiments also revealed consistently higher Tiam1 levels in 
a
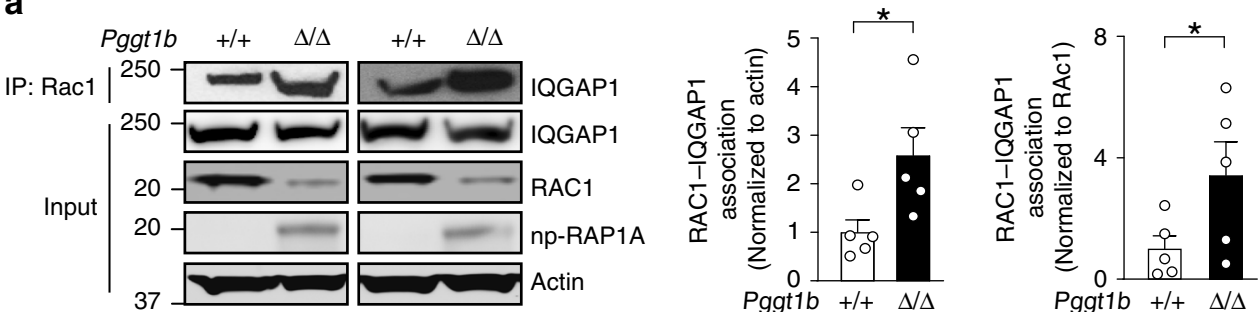

b

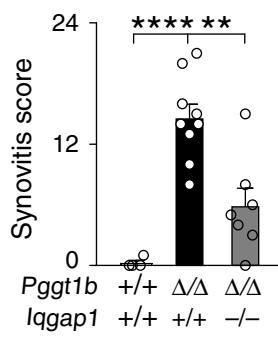

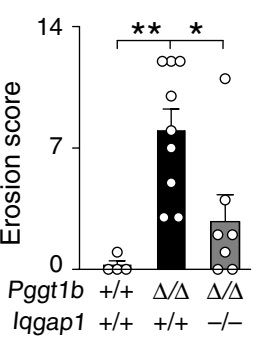
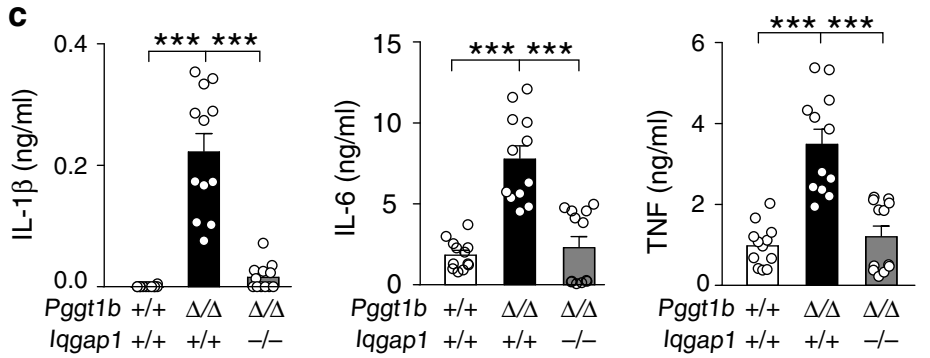

d
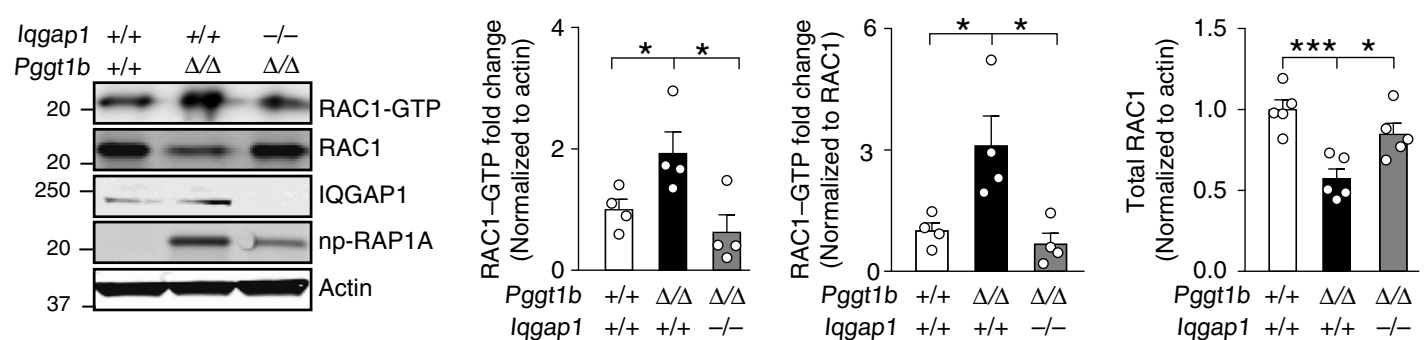

e

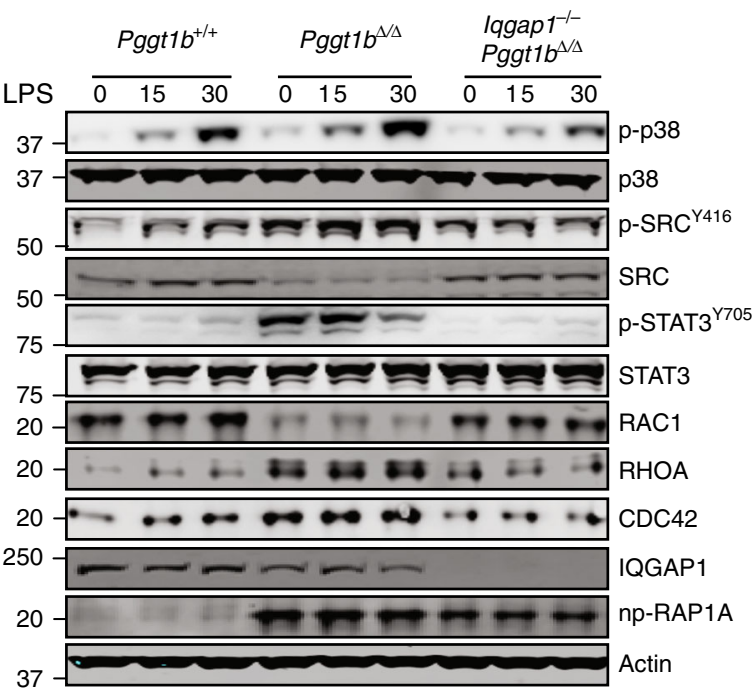

f
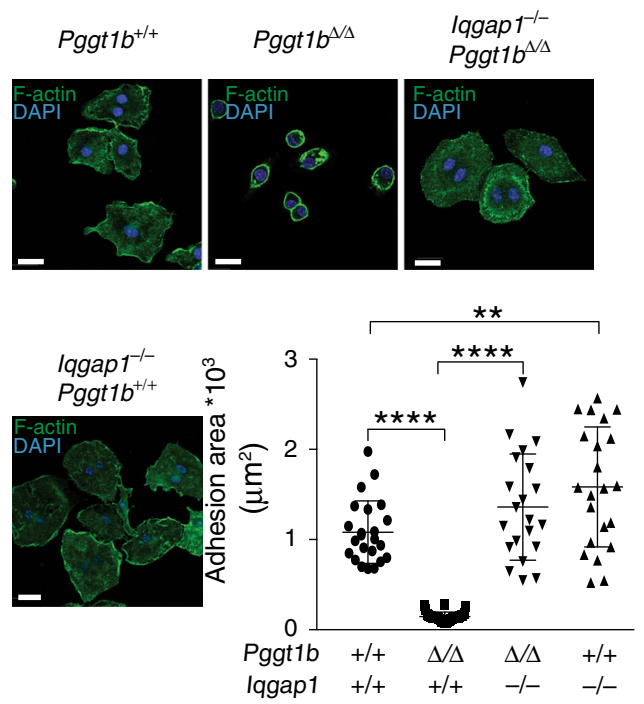

Fig. 3 lqgap1 binds nonprenylated Rac1 and mediates GTP loading, cytokine production, and arthritis. a Left, immunoprecipitation (IP) of Rac1 in BM macrophage lysates followed by western blots for lqgap1. Direct western blots were performed on the same lysates (Input) to quantify total lagap1 and Rac1 levels. Nonprenylated Rap1A was used as marker of GGTase-I-deficient cells; actin was used as a loading control. Middle and right, levels of Rac1bound lqgap1 was determined by densitometry of IP-western blots of BM macrophages from six mice/genotype. $\mathbf{b}$ Synovitis and erosion scores in joints of

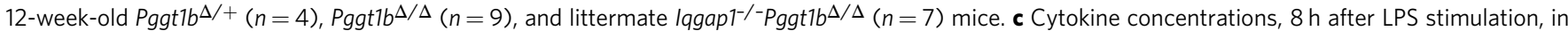
medium of BM macrophages isolated from Pggt $7 b^{\Delta /+}, P g g t 1 b^{\Delta / \Delta}$, and lqgap $1^{-/-P g g t} 7 b^{\Delta / \Delta}$ mice $(n=3 /$ genotype). $\mathbf{d}$ Left panel, Western blots showing

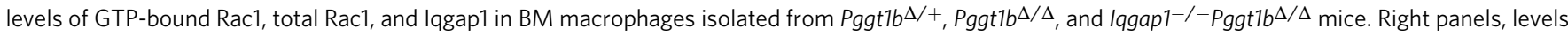
of GTP-bound and total Rac1 determined by densitometry $(n=5 /$ genotype). e Western blots of macrophage lysates isolated at base-line and 15 and 30 min after LPS stimulation. f Confocal microscopy images showing F-Actin staining of BM macrophages. Scale bars: $10 \mu \mathrm{m}$. Error bars represent s.e.m. The graph shows adhesive area of 20-30 macrophages in ten randomly selected fields analyzed in cells from two mice/genotype. Significance between groups were calculated with two tailed Student's $t$ test $(\mathbf{a}, \mathbf{c}, \mathbf{d}, \mathbf{f})$ and one-way ANOVA with Tukey's post hoc test (b). n.s. not significant, ${ }^{\star} P<0.05,{ }^{\star \star} P<0.01$, ${ }^{\star \star \star} P<0.001$ 

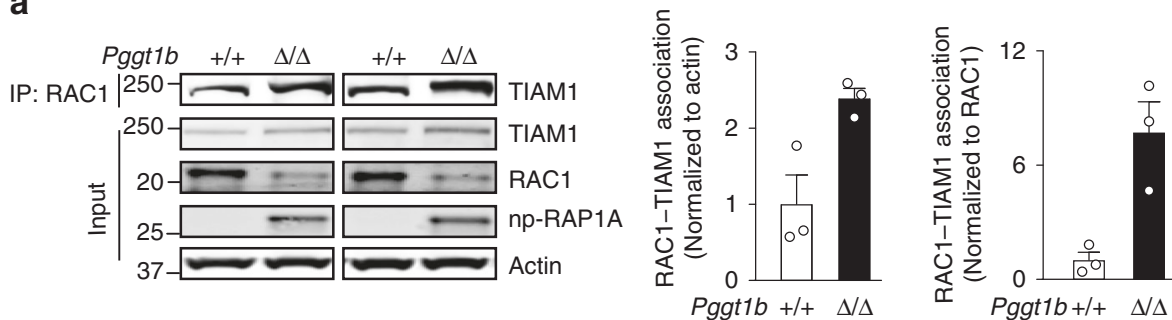

b
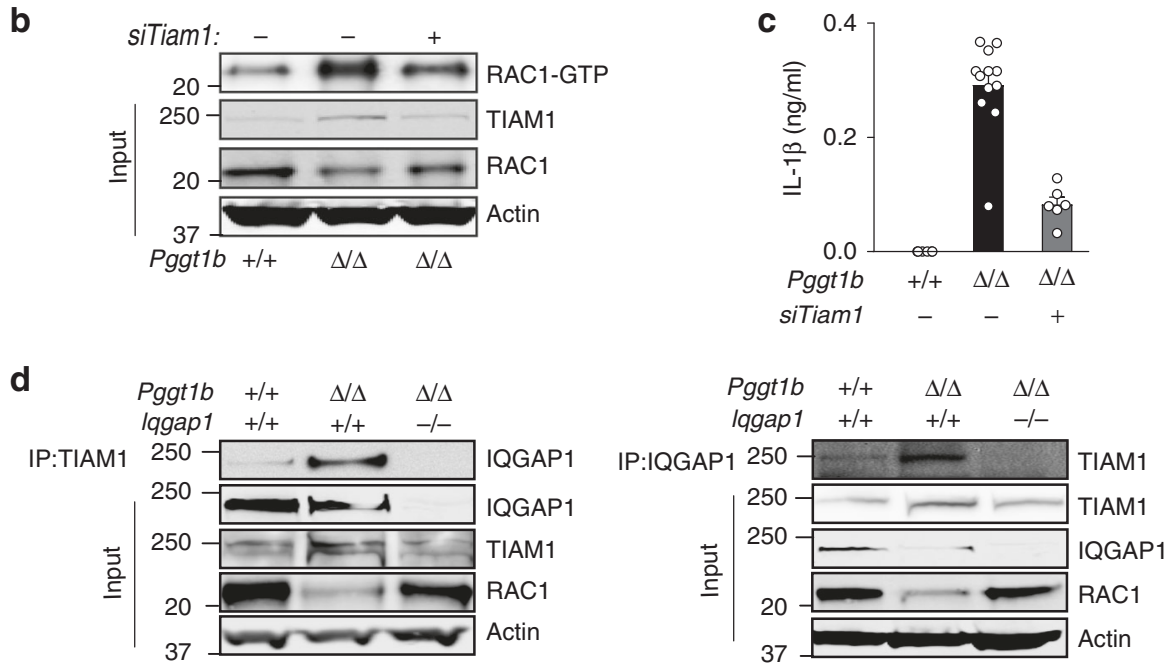

Fig. 4 Tiam1 binds nonprenylated Rac1 and supports GTP loading and II-1 $\beta$ production. a Left, immunoprecipitation (IP) of Rac1 in BM macrophage lysates followed by western blots for Tiam1. Direct western blots were performed on the same lysates (Input) to quantify total Tiam1 and Rac1 levels. Middle and right, levels of Rac1-bound Tiam1 was determined by densitometry of IP-western blots ( $n=3 /$ genotype). Actin was the loading control. b Western blot showing levels of GTP-bound Rac1 in lysates of BM macrophages pre-incubated for $24 \mathrm{~h}$ with scrambled or Tiam1-targeted siRNAs. Direct western blots were performed on the same lysates (Input) to quantify total Tiam1 and Rac1 levels. c Concentration of II-1 $\beta, 8 \mathrm{~h}$ after LPS stimulation, in medium of BM macrophages pre-incubated for $24 \mathrm{~h}$ with scrambled or Tiam1-targeted siRNAs ( $n=2$ genotype). d Left, IP of Tiam1 followed by western blot for lagap1. Right, IP of lagap1 followed by western blot for Tiam1. Direct western blots were performed on the same lysates (Input) to quantify total lagap1, Tiam1, and Actin levels. Error bars represent s.e.m. Significance between groups were calculated with two tailed Student's $t$ test. ${ }^{\star} P<0.05$

$\operatorname{Pggt}_{1} b^{\Delta / \Delta}$ than in control macrophages (Fig. 4a, b, d); the Iqgap1 knockout normalized Tiam1 levels (Fig. 4d).

Editing the Rac1 CAAX sequence increases GTP loading. Levels of GTP-bound and total Rac1 in GGTase-I-deficient cells could conceivably be influenced by the accumulation of other nonprenylated $C A A X$-proteins. To define biochemical consequences of blocking Racl prenylation in cells expressing normal GGTase-I activity, we edited the CAAX sequence of endogenous Racl in human embryonic kidney (HEK) cells with a nonintegrating homologous recombination-based CRISPR/CAS9 approach. We isolated three $C A A X$-mutant clones (Rac1CM1-3), sequenced their DNA and cDNA (Fig. 5a), and found in their lysates that Racl exhibited a reduced electrophoretic mobility indicating that the protein had not been prenylated by GGTase-I (Fig. 5b). Rac1GTP levels were two to fourfold higher in $C A A X$-mutant than in control cells; and Racl total levels were two to threefold lower (Fig. 5c). Further analyses revealed that the distribution of Rac1 in cytosol and membrane fractions was similar in CAAX-mutant and control cells (Fig. 5d). However, nuclear Racl levels were lower in the edited cells (Fig. 5d). Similar to the findings with $P_{g g t} 1 b^{\Delta / \Delta}$ macrophages, Rac1 total levels increased following lactacystin and MG-132 administration (Fig. 5e, f). Moreover, Racl ubiquitination and Iqgapl association was higher in Rac1 mutant than control cells (Fig. $5 \mathrm{~g}, \mathrm{~h}$ ).
Statins mimic effects of GGTase-I deficiency. To determine whether statins can produce similar cellular effects as GGTase-I deficiency, we incubated three different macrophage types with Atorvastatin, Rosuvastatin, and Simvastatin. Statins increased Rac1-GTP levels and reduced total Rac1; and increased GTPbound and total RhoA (Fig. 6a, b). Moreover, statins facilitated Il$1 \beta$ maturation and secretion in response to LPS, and potentiated Il-6 and Tnf production (Fig. 6c, d, and Supplementary Fig. 7A-C). Pre-incubating the macrophages with GGPP abolished the statin effect on cytokine production in most, but not all cases (Fig. 6c, d, and Supplementary Fig. 7A and B). To determine if Iqgap1 underlies the ability of statins to increase cytokine production, we incubated primary Iqgap1-knockout macrophages with Simvastatin and found that LPS-stimulated cytokine production was either reduced or normalized (Fig. 6e). Inhibiting Iqgap1 with siRNAs produced similar results (Supplementary Fig. 7D).

\section{Discussion}

In this study, we identified three components of the mechanism underlying inflammatory phenotypes of mice with macrophagespecific GGTase-I deficiency. First, GGTase-I prenylates at least 60 substrates $^{17}$, and our data indicate that one of them, Rac1, mediates the majority of the robust innate immune responses. Second, nonprenylated Racl becomes hyperactivated through an 
a
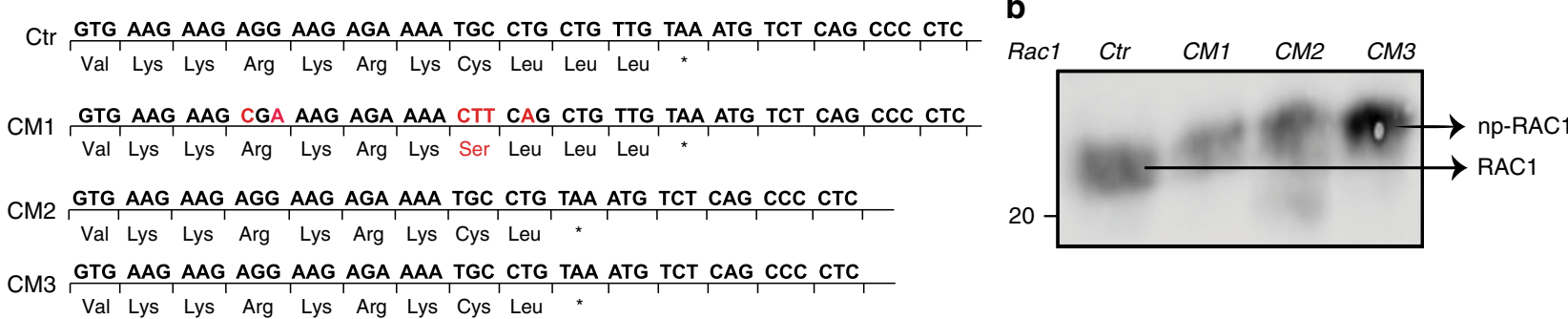

C

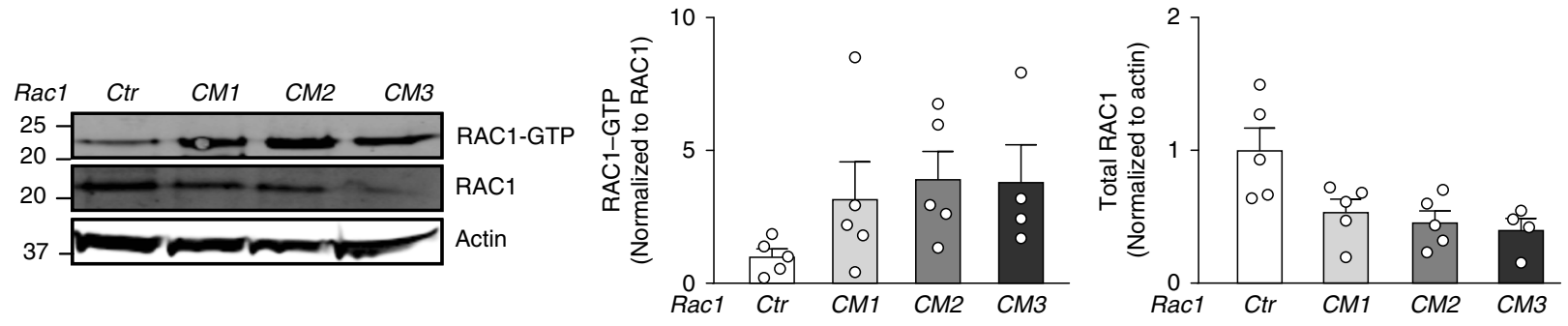

d
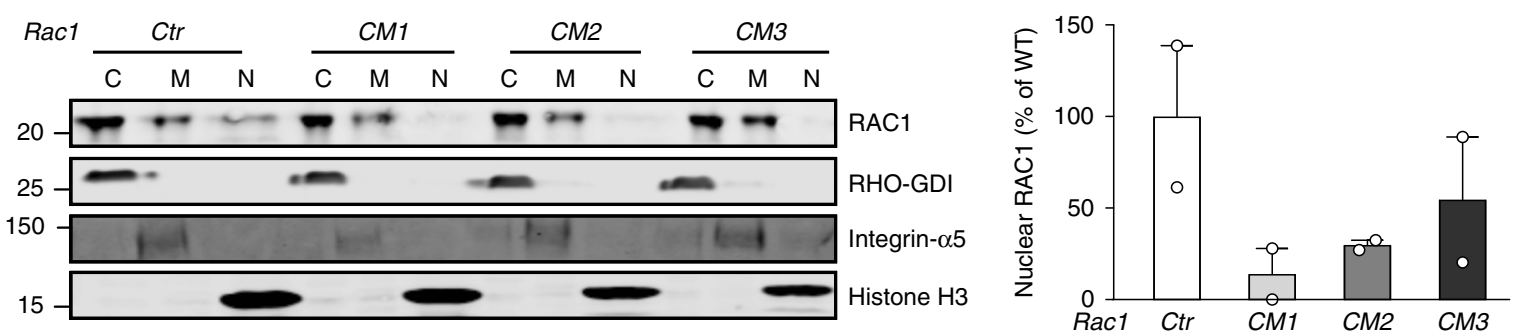

e

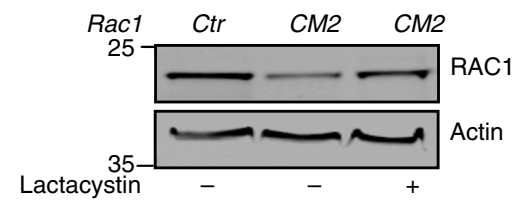

f

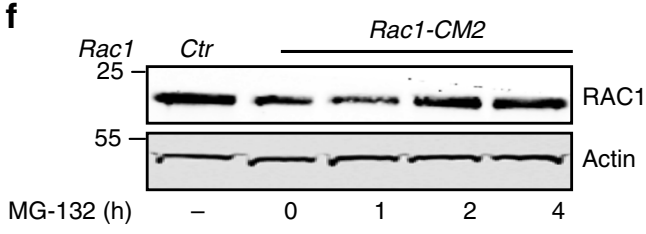

g
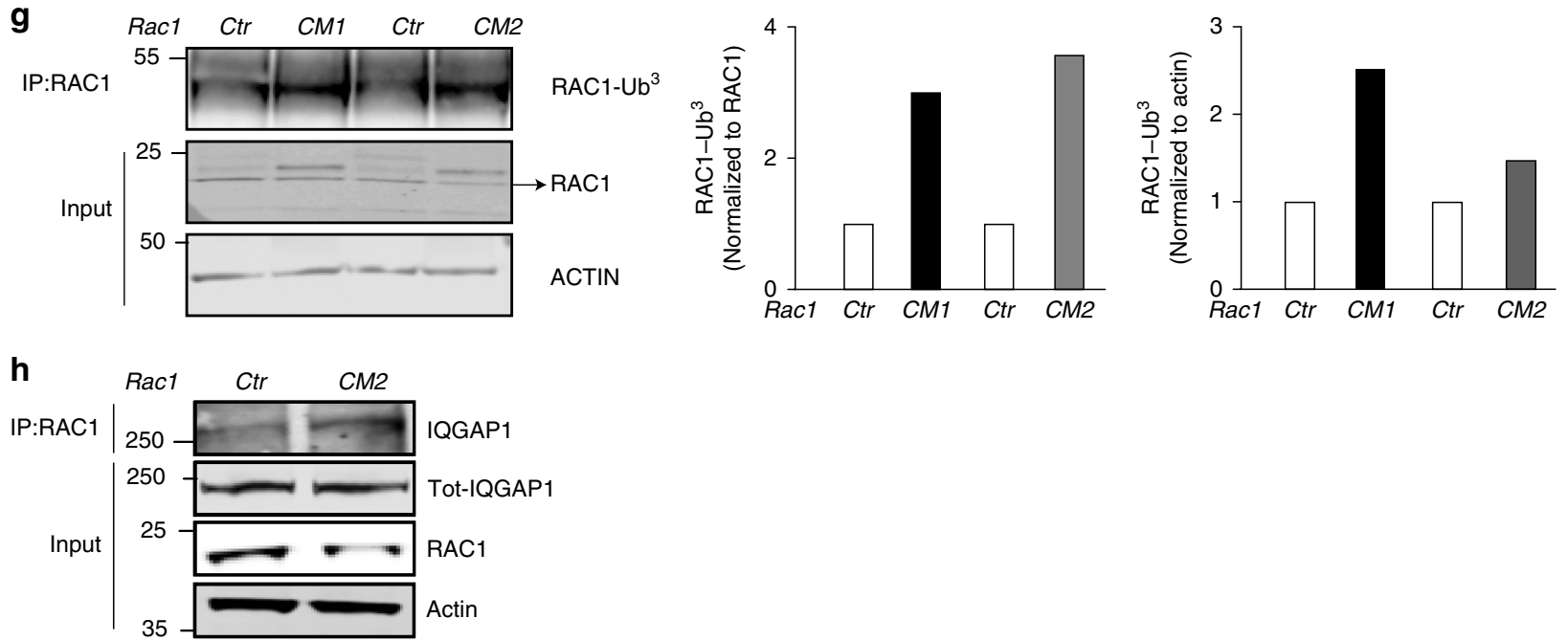

increased interaction with Tiam1 and Iqgap1. And third, hyperactive Rac1 activates inflammasome-, ROS-, and p38-driven signaling pathways that enhance LPS-induced Il-1 $\beta$, Il-6, and Tnf production.

Prenylation is believed to have evolved as a strategy to target proteins to membranes, promote effector interactions, and thereby stimulate activation and signaling ${ }^{34}$. Several results indicate that this is not the case for Rac1. First, Rac1 membrane/ cytosol partitioning was unaffected in GGTase-I-deficient macrophages ${ }^{14}$ and in HEK cells engineered to express endogenous Racl with $C A A X$ motif mutations. Second, interactions between nonprenylated Rac1 and the effectors Tiam1 and Iqgap1 were substantially increased. And third, GTP-binding was increased and downstream signaling pathways were activated. The simplest 
Fig. 5 Editing the endogenous Rac1 CAAX-motif increases GTP loading and reduces total Rac1 levels. a Predicted amino acid sequence from sanger sequence results of PCR-amplified Rac1 DNA and cDNA fragments. The DNA/cDNA was from HEK-293 clones whose Rac1 gene had been edited at the CAAX sequence by CRISPR/Cas9-facilitated homologous recombination to prevent geranylgeranylation by GGTase-I. CM1-3, CAAX mutant clones; Ctr, unedited control clone. b Left, western blot showing electrophoretic mobility of wild-type Rac1 in Rac1Ctr lysates, and nonprenylated (np) Rac1 in CM1-3 lysates. c Left, western blots showing amounts of GTP-bound Rac1, total Rac1, and the loading control Actin in lysates of the gene-edited cells. Middle and right, levels of GTP-bound and total Rac1 determined by densitometry; data are mean of three independent experiments for each cell line. $\mathbf{d}$ Left, western blots showing the distribution of Rac1 in cytosol, membrane, and nuclear fractions (designated $\mathrm{C}, \mathrm{M}$, and N, respectively) of the gene-edited cells. RhoGDI1, integrin- $\alpha 5$, and histone H3 were used as markers for cytosol, membrane, and nuclear fractions, respectively. Right, levels of nuclear Rac1 determined by densitometry data of three independent experiments and expressed as percent of Rac1Ctr. e, $\mathbf{f}$ Western blots showing levels of total Rac1 and the loading control Actin in lysates of gene-edited cells isolated after incubation with $15 \mu \mathrm{M}$ lactacystin for $12 \mathrm{~h}$ (e); and $50 \mu \mathrm{M}$ MG-132 at different time points (f). $\mathbf{g}$ Left, immunoprecipitation (IP) of Rac1 in gene-edited cells followed by western blot for Ubiquitin. Direct western blots were performed on the same lysates (Input) to quantify total Rac1 levels. Actin was used as a loading control. Right, Bar graphs showing levels of Rac1-Ub ${ }^{3}$. h Immunoprecipitation (IP) of Rac1 in gene-edited cells followed by western blot for lqgap1. Direct western blots were performed on the same lysates (Input) to quantify lqgap1 and Rac1 levels. Actin was used as a loading control. Error bars represent s.e.m

explanation for these results is that GGTase-I-mediated prenylation normally acts as a break on innate immune responses in macrophages by limiting Racl effector interactions. Blocking prenylation releases the break, stimulates Racl interactions, and unleashes wide-spread proinflammatory signaling (Supplementary Fig. 8).

This reasoning is particularly relevant for the control of Il-1 $\beta$ production. Due to its potent pleiotropic inflammatory effects, Il$1 \beta$ secretion by macrophages is tightly controlled and requires two different events: a priming step through cell-surface receptors that leads to $I l 1 b$ transcription; and a second signal that activates caspase- 1 and leads to pro-Il-1 $\beta$ cleavage and secretion of the mature protein 35,36 . Our data suggest that nonprenylated Rac1 can act as the second signal in Il-1 $\beta$ production. This argument is supported by the finding that the robust caspase-1-mediated Il- $1 \beta$ production in GGTase-I-deficient macrophages was abolished by normalizing Rac1-GTP levels-which was accomplished by knocking out one copy of Rac1 or both copies of Iqgap1.

GGTase-I deficiency increased basal phosphorylation of Src, Stat 3 , and Ikka/ $\beta$ - and to some extent p38-in a Rac1-dependent fashion; LPS-induced phosphorylation of all four proteins was also increased. Rac1-induced activation of Src, Stat3, and Ikk was mediated by ROS, whereas p38 activation was likely a direct consequence of Rac1/PAK activity. These findings are consistent with previous reports that Racl triggers ROS production by NADPH oxidases which activates Stat $3^{37,38}$; although other studies show a physical interaction between Rac1-GTP and Stat $3^{39}$. Interestingly, Stat 3 contributes to the progression of chronic inflammation and joint destruction in mouse models of rheumatoid arthritis ${ }^{40,41}$. Src and p38 are also involved in Ikkdependent activation of NFKB which stimulates transcription of cytokines including Il-1 $\beta$, Il-6, and $\mathrm{Tnf}^{42-44}$. Importantly, knockout of one copy of Rac1 normalized Rac1-GTP levels in GGTase-I-deficient macrophages and reduced or normalized signaling of the entire pathway.

Knockout of Iqgap1 normalized Rac1-GTP loading, abolished proinflammatory signaling and cytokine production, and markedly reduced arthritis in GGTase-I-deficient mice. Although it is not known whether Iqgap1 encounters nonprenylated Rho proteins in activated wild-type macrophages, it is possible that targeting Iqgap1 might be useful in the therapy of some inflammatory conditions. One example would be patients with mevalonate kinase deficiency (MKD) - an autoinflammatory disease associated with high Il-1 $\beta$ production, fever episodes, lymph node enlargment, and joint pain. MKD leads to reduced synthesis of GGPP which reduces prenylation ${ }^{23,45-47}$, and could therefore enhance cytokine production through an increased Rac1-Iqgap1 interaction. Targeting Iqgap1 would likely be associated with few side-effects as Iqgap1-deficient mice are viable and exhibit only mild phenotypes late in life $\mathrm{e}^{33}$; and their macrophages responded normally to LPS. Targeting Rac1 might be an alternative strategy and has been proposed earlier ${ }^{23,46}$; but Rac1 deficiency is lethal in mice and produces a range of cellular and tissue phenotypes.

The knockout of Iqgap1 rescued most of the robust proinflammatory phenotypes of GGTase-I-deficient macrophages but did not influence LPS-induced cytokine production of GGTase-I wild-type macrophages-despite reducing Rac1-GTP levels. The simplest explanation for these observations is that both the levels of Rac1-GTP and the affinity of Racl for Iqgap1 were markedly higher in GGTase-deficient than wild-type macrophages; thus Iqgapl's role in controlling Rac1-GTP levels and its downstream signaling could simply be comparatively more important in the GGTase-I-deficient cells. Whether or not Iqgap1 influences Rac1GTP signaling and cytokine production in arthritis and other inflammatory diseases in the setting of wild-type GGTase-I remains to be determined.

Statin administration produced similar effects as the knockout of GGTase-I: Rac1-GTP levels increased, as did LPS-induced cytokine production, in a GGPP-dependent fashion. Part of those results are in line with previous studies ${ }^{23,48}$, but here we also provide evidence that the proinflammatory statin effect requires Iqgap1. It, therefore, seems reasonable to speculate that statins' pro-inflammatory effects on macrophages stem from reduced Rac1 prenylation and increased interaction with Iqgap1. However, statin therapy is more often associated with antiinflammatory than pro-inflammatory effects. A potential explanation is that anti-inflammatory statin effects are the result of the drug's action on lymphocytes rather than macrophages. For example, statins inhibit T-cell proliferation and differentiation, and immune synapse formation ${ }^{12}$. Another speculation would be that some side-effects of statin therapy, such as myositis and rhabdomyolysis, are caused by hyperactivation of a nonprenylated Rho protein. However, these speculations should be interpreted with caution because there is little evidence that statins inhibit prenylation in vivo. Daily statin doses used by patients range from 5 to $80 \mathrm{mg} /$ day, resulting in plasma concentrations of $1-15 \mathrm{nM}^{49}$. The doses used in vitro in the present study (i.e., $1-5 \mu \mathrm{M}$ ) are lower than those of many other stu$\operatorname{dies}^{50-52}$, but they are likely higher than those that cells in vivo are exposed to.

Our results show clearly that prenylation is not required for GTP loading and activation of Rho proteins. Prenylation actually inhibits GTP loading. Why then are Rho proteins prenylated? One potential role of prenylation is to fine-tune the targeting of Rho proteins to specific subcellular membrane domains. Another possibility is that prenylation is required for rapid cycling between GTP and GDP-bound states during specific functions of 
a
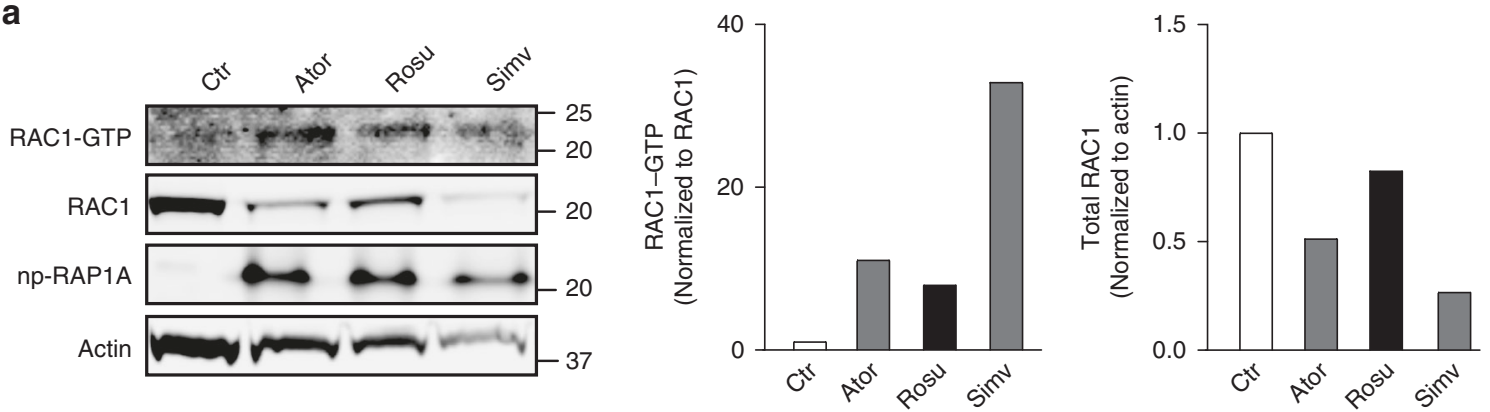

b
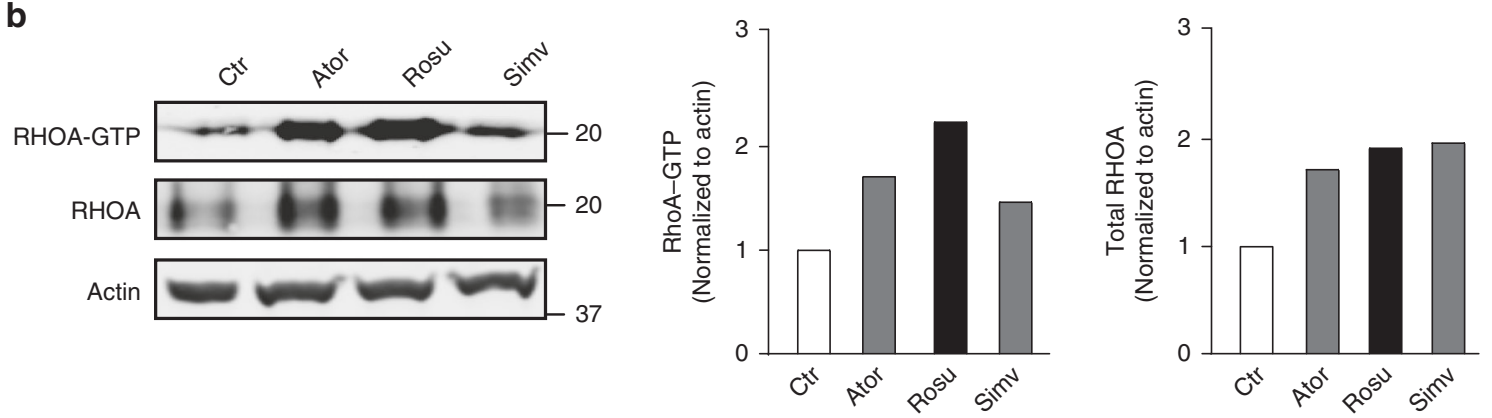

C

RAW264 macrophages

d
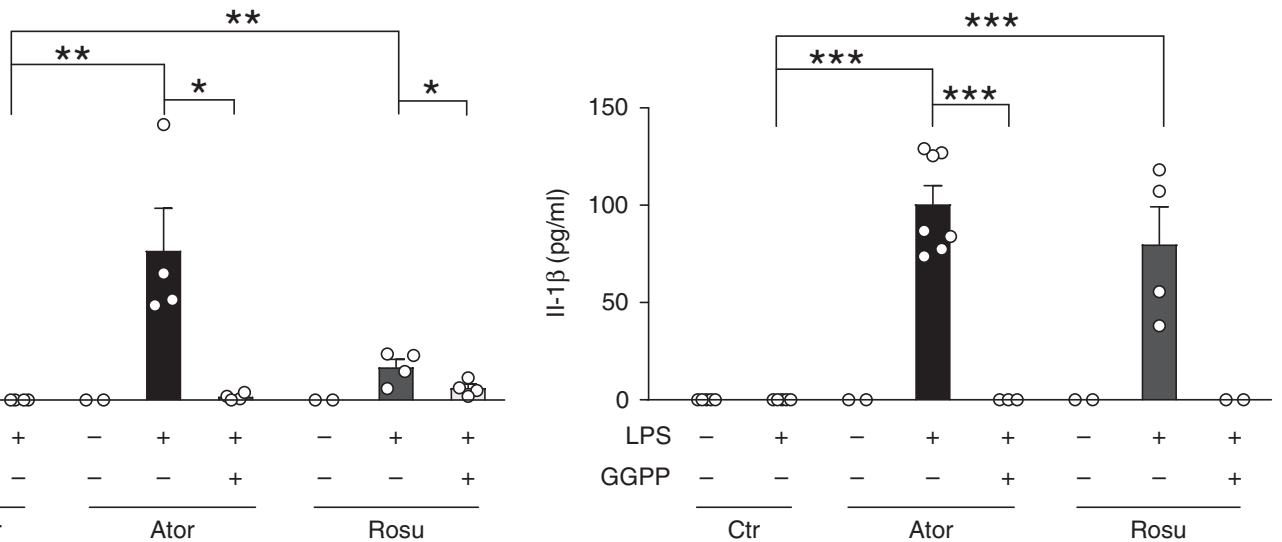

e

Primary mouse macrophages
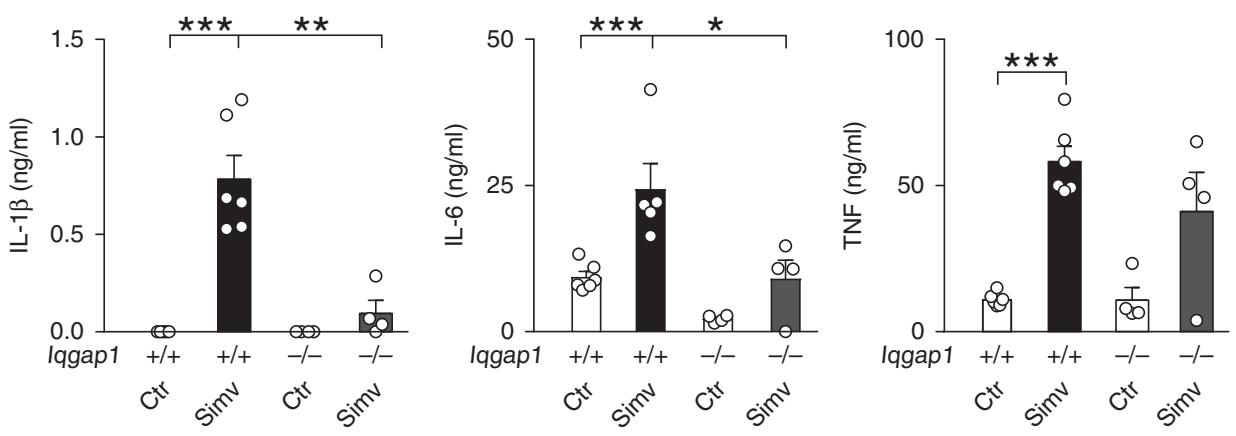

Fig. 6 Statins increase Rac1-GTP and cytokine production in a GGPP- and lqgap1-dependent fashion. a Left, western blots showing levels of GTP-bound and total Rac1 in lysates of RAW 264.7 macrophages incubated for 3 weeks with Atorvastatin $(5 \mu M)$, Rosuvastatin $(2.5 \mu M)$, and Simvastatin $(1 \mu M)$. NpRap1A was used as a marker of GGTase-I-deficient cells and Actin as a loading control. Middle and right, amounts of GTP-bound and total Rac1 determined by densitometry. b Left, western blots showing levels of GTP-bound and total RhoA in the same cells as in (a). Middle and right, amounts of GTP-bound and total RhoA determined by densitometry. c II-1 $\beta$ concentration, before and $8 \mathrm{~h}$ after LPS stimulation, in medium of RAW 264.7 macrophages incubated with Atorvastatin $(5 \mu \mathrm{M})$ and Rosuvastatin $(2.5 \mu \mathrm{M})$ for 21 days. GGPP $(10 \mu \mathrm{M})$ was added to the cells 3 days before LPS stimulation. $\mathbf{d}$ Similar experiment as in c performed with $\mathbf{5 7 4}$ macrophages. e Cytokine concentration in medium of LPS-stimulated lqgap $1^{+/+}$and lqgap1 ${ }^{-/-}$macrophages incubated with Simvastatin $(5 \mu \mathrm{M})$ for $60 \mathrm{~h}$. Error bars represent s.e.m. Significance between groups were calculated with two tailed Student's $t$ test. ${ }^{\star} P<0.05$, ${ }^{\star \star} P<0.01$, ${ }^{\star \star \star} P<0.001$ 
the cell such as during proliferation or certain types of cell movement. These issues will be possible to address in the future.

But based on the current study, we conclude that a major role of GGTase-I-mediated prenylation in macrophages is to limit Rac1 activation and pro-inflammatory signaling, prevent Rac1dependent Il-1 $\beta$ maturation, and thereby restrain innate immune responses.

\section{Methods}

Mouse breeding and genotyping. Mice harboring conditional knockout alleles of the beta subunit of GGTase-I (Pggt $\left.1 b^{\mathrm{fl} / \mathrm{fl}}\right)$ were bred with lysozyme M-Cre (LC) knock-in mice to produce offspring lacking GGTase-I in macrophages as described $^{6}$; these mice were designated $\operatorname{Pggt} 1 b^{\Delta / \Delta}\left(\Delta=\right.$ delta, deleted allele). Pggt $1 b^{\Delta / \Delta}$ mice were bred with mice harboring conditional knockout alleles of Racl, Rhoa and $C d c 42$, and conventional knockout alleles for Iqgap $1^{33,53-55}$. Genotyping was performed by polymerase chain reaction (PCR) on genomic DNA from ear or tail biopsies $^{6}$. Mice were housed in a specific pathogen-free facility monitored by routine testing of sentinel mice, and were given free access to water and chow. Mice were on a mixed genetic background (1290la/Hsd-C57BL/6) and control mice in all experiments were gender-matched littermates. Animal experiments were approved by the research animal ethics committee in Gothenburg, Sweden.

Histology and quantification of arthritis in joints. Joints from 12-week-old mice were fixed in $4 \%$ paraformaldehyde and stored in $70 \%$ ethanol. The joints were decalcified, embedded in paraffin, sectioned, and stained with hematoxylin and eosin. The slides were analyzed in a Zeiss Axioplan 2 microscope (Carl Zeiss AG). Synovitis and erosion scores were evaluated in sections of knee, ankle, metatarsal, elbow, wrist, and metacarpal joints by an observer blinded to genotype. An arbitrary scale from 0 to 3 was used: 0 -is a healthy joint, 1 -hypertrophy/mild proliferation of synovia constituting more than two intimal lining cell layers, and mononuclear cell infiltration is visible; 2 -hypercellularity, multiple inflammatory foci in the sub-lining layer, and proliferation of synovia; and 3-massive influx of inflammatory cells scattered throughout the synovial tissue, and growth of granulation tissue (pannus). Cartilage and bone erosion were evaluated with a separate $0-3$ scale: 0 is a healthy joint; 1 -reduction or uneven cartilage thickness; 2 -compromised cartilage integrity and formation of erosions on the cartilage surface under the growing pannus; and 3-loss of articular surface, obliteration of the joint cavity, and loss of joint or bone shape. The cumulative arthritis index for each mouse was constructed by adding the scores from the fore and hind paws ${ }^{56}$.

\section{Isolating and culturing bone marrow macrophages. Bone marrow cells were} isolated from femur and tibia and cultured for 7-10 days in DMEM high glucose medium $(61,965,059)$ supplemented with $10 \%$ fetal bovine serum $(10270-106$, Thermo Fisher Scientific), 1\% HEPES (H0887, Sigma-Aldrich), 1\% glutamine (929070, Thermo Fisher Scientific), 1\% gentamycin (11482524, Fischer Scientific), $0.01 \% \beta$-mercaptoethanol, and 10\% CMG14-12 cell supernatant as a source of macrophage colony-stimulating factor (M-CSF $)^{57}$.

Protein analyses. Rac1-GTP levels were assessed with the Active Racl Pulldown and Detection kit (16118, Thermo Fisher Scientific); Cdc42-GTP with the Active Cdc42 Pulldown and Detection kit (16119, Thermo Fisher Scientific); and RhoAGTP with the RhoA Activation Assay Biochem Kit (BK036, Cytoskeleton). For IP, cells were lysed in a buffer containing $25 \mathrm{mM}$ Tris- $\mathrm{HCl}$ pH 7.4, $150 \mathrm{mM} \mathrm{NaCl}, 1$ mM EDTA, 1\% NP-40, and 5\% glycerol; and IP was performed with antibodies recognizing Rac1 (05-389, Millipore); Cdc42 (sc-87), Iqgap1 (sc-10792), and Tiam1 (sc-376021, Santa Cruz Biotechnology); and RhoA (ARH03, Cytoskeleton), using the Dynabeads Protein G Immunoprecipitation Kit (10007D, Thermo Fisher Scientific). Ubiquitinated Racl was isolated with the Pierce Ubiquitin Enrichment Kit (89899, Thermo Fisher Scientific). Subcellular fractions were isolated with the Qproteome Cell Compartment Kit (37502, Qiagen). Detergent and aqueous fractions were isolated with TX-114 assays as described ${ }^{58}$. To quantify Racl turnover rates, cell lysates were isolated from macrophages after incubation with cycloheximide $(15 \mu \mathrm{g} / \mathrm{ml})$ to stop protein synthesis. Western blots were performed by loading equal amounts of total proteins from whole-cell lysates or cellular fractions on Bolt $4-12 \%$ Bis-Tris gels (Thermo Fisher Scientific) and 18\% sodium dodecyl sulphate polyacrylamide gel electrophoresis. Proteins were transferred to nitrocellulose membranes which were incubated with antibodies to Racl (05-389, Millipore); ACTIN (A1978, Sigma-Aldrich); Mmp13 (sc-30073), nonprenylated RAP1A (sc-1482), RacGAP1 (sc-98617, Santa Cruz Biotechnology); Iqgap1 (SC 376021, Santa Cruz Biotechnology), Tiaml (A300-099A, Bethyl Laboratories); Histone-H3 (ab18521, Abcam); RhoA (2117S), Cdc42 (2462S), phospho-p38 (9211S), p38 (9212S), phospho-Stat3 ${ }^{\mathrm{Y} 705}(9145 \mathrm{~S})$, Stat3 (9132S), phospho-Src ${ }^{\mathrm{Y} 416}$

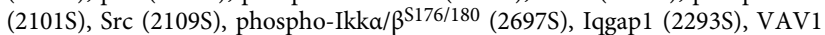
(2502S), VAV2 (2848S), DOCK180 (4846S), Integrin- $\alpha 5$ (4705S), and $\beta$-PIX (4515S, Cell Signaling Technology); Il-1 $\beta$ (AF-401-NA, R\&D System); and anticaspase-1 (p20) (AG-20B-0042-C100, Adipogen). Protein bands were visualized with infrared dye-conjugated secondary anti-mouse (926-32212), anti-rabbit
(926-32,211), and anti-goat (926-32,214, LI-COR) antibodies and analyzed in a LICOR Odyssey Imager. Band densities were analyzed with Image J. Mmp13 in supernatants of LPS-stimulated macrophages was analyzed by western blots using Mmp13 antibodies (sc-30073, Santa Cruz Biotechnology) and horseradishconjugated anti-rabbit (NA934, GE Healthcare Lifesciences) antibodies and the ECL western blotting system (RPN2232, GE Healthcare Lifesciences), as described $^{59}$. Primary antibodies were used at 1:500 dilution, except anti-Mmp13 and -TIAM1 which were used at 1:250 dilution.

In vitro prenylation assay. Racl prenylation was detected with a click chemistry approach $^{60}$. Macrophages were incubated for $48 \mathrm{~h}$ with $30 \mu \mathrm{M}$ Click-IT Geranylgeranyl Alcohol, Azide, mixed isomers (C10249, Thermo Fisher Scientific). Cells were then lysed in a buffer containing $25 \mathrm{mM}$ Tris- $\mathrm{HCl}, 150 \mathrm{mM} \mathrm{NaCl}, 1 \mathrm{mM}$ EDTA, $1 \%$ NP-40, and 5\% glycerol, supplemented with protease and phosphatase inhibitors. IP was performed with Rac1 antibodies (05-389, Millipore) using the Dynabeads Protein G Immunoprecipitation Kit (10007D, Thermo Fisher Scientific). The click chemistry reaction was performed on the immunoprecipitate with a buffer containing $10 \mu \mathrm{M}$ Alexa Fluor 488-alkyne (A10267, Thermo Fisher Scientific), $1 \mathrm{mM}$ tris(2-carboxyethyl)phosphine (TCEP), $100 \mu \mathrm{M}$ tris[(1-benzyl-1H1,2,3-triazol-4-yl)methyl]amine (TBTA), and $1 \mathrm{mM} \mathrm{CuSO} 4$ in PBS for $1 \mathrm{~h}$. The immunoprecipitates were washed three times with PBS containing $1 \%$ NP-40; eluted in LDS sample buffer; and then the proteins were resolved on $4-12 \%$ gels. Fluorescent (i.e., prenylated) Racl in gels was detected by Gel Doc $\mathrm{XR}^{+}$molecular imager (BioRad)

Rac1 ubiquitination. Ubiquitinated Rac1 was detected as described ${ }^{61}$. HEK293 cells were lysed in a buffer containing $2 \%$ sodium deoxycholate, $150 \mathrm{mM} \mathrm{NaCl}, 10$ $\mathrm{mM}$ Tris- $\mathrm{HCl}$, and supplemented with $10 \mu \mathrm{M}$ MG132, $10 \mu \mathrm{M}$ PR619, protease and phosphatase inhibitors. Lysates were boiled for $10 \mathrm{~min}$; sonicated; diluted ten times in a buffer containing $10 \mathrm{mM}$ Tris- $\mathrm{HCl}, 150 \mathrm{mM} \mathrm{NaCl}, 2 \mathrm{mM}$ EDTA, and $1 \%$ Triton; incubated at $+4{ }^{\circ} \mathrm{C}$ for $1 \mathrm{~h}$ with continuous rotation; and then clarified by centrifugation at $20,000 \times g$ for $30 \mathrm{~min}$. IP was performed with Racl antibodies (05389, Millipore) using the Dynabeads Protein G Immunoprecipitation Kit (10007D, Thermo Fisher Scientific). Western blots were performed on Bolt $4-12 \%$ Bis-Tris gels (Thermo Fisher Scientific). Proteins were transferred to nitrocellulose for western blots with Ubiquitin antibodies (Thermo Fischer Scientific).

Gene-expression analyses. RNA was isolated from macrophages with the RNeasy Mini kit (74104, Qiagen) $8 \mathrm{~h}$ after LPS stimulation (tlrl-eblps, Invivogen). Complementary (c) DNA was synthesized from RNA with the iScript cDNA synthesis kit (1708890, Biorad). Quantitative real-time PCR was performed with Taqman assays for mouse Rac1 (Mm01201657_g1), Rhoa (Mm00834507_g1), Cdc42 (Mm01194005_g1), Tiam1 (Mm00437079_m1), and Actb (4352933E, Thermo Fisher Scientific); and with SYBR-green using primers listed in Supplementary Table 1; in a 7900HT-fast machine (Applied Biosystems).

Cytokine analyses and inhibitors. Macrophages were cultured overnight in medium without M-CSF and stimulated with LPS $(10 \mathrm{ng} / \mathrm{ml})$ in fresh medium. In some experiments the macrophages were incubated for $30 \mathrm{~min}$ with a p38 inhibitor (SB203580, Invivogen) and a ROS inhibitor (D2926, Sigma-Aldrich) before LPS stimulation; in other experiments the macrophages were incubated for 24-48 h with small-interfering (si) RNAs targeting Arhgdia (AM16706, Thermo Fischer Scietific), Tiam1 (D-047808-03-0050), Iqgap1 (D-040589-01-0050), or containing a scrambled sequence (D-001206-14-50, Dharmacon) before LPS stimulation. For statin experiments, macrophages were treated for $60 \mathrm{~h}$ or 18 days with Atorvastatin, (PZ0001, Sigma Aldrich), Rosuvastatin (SML1264, Sigma Aldrich) and Simvastatin (S6196, Sigma Aldrich). GGPP ammonium salt (G6025, Sigma Aldrich) was added to the cells 3 days before LPS stimulation. Supernatants were collected before and $8 \mathrm{~h}$ after LPS stimulation and levels of Tnf, Il-6, and Il-1 $\beta$ were determined by ELISA (88-7324-76, 88-7064-76, and 88-7013-76, respectively, eBioscience).

Mass spectrometry analysis of Rac1-interacting proteins. Macrophages were lysed in buffer containing $25 \mathrm{mM}$ Tris- $\mathrm{HCl}, 150 \mathrm{mM} \mathrm{NaCl}, 1 \mathrm{mM}$ EDTA, 5\% glycerin, and 1\% CHAPS. Rac1 was immunoprecipitated with the 05-389 antibody (Millipore) using the Dynabeads Protein G Immunoprecipitation Kit (10007D, Thermo Fisher Scientific). The protein complex was eluted with $50 \mathrm{mM}$ triethylammonium bicarbonate and 4\% SDS, and digested with trypsin using the filteraided sample preparation method, as described ${ }^{30}$. The digested peptides were labeled with 10-plex isobaric tandem mass tag (TMT) reagents (Thermo Scientific). The labeled samples were combined into one TMT-set; purified with trifluoroacetic acid precipitation and HiPPR Detergent Removal Resin (Thermo Scientific). The purified sample was fractionated into twelve fractions using the Pierce High $\mathrm{pH}$ Reversed-Phase Peptide Fractionation Kit (Thermo Scientific), and the fractions were dried in a vacuum centrifuge and reconstituted in $20 \mu \mathrm{l}$ of $3 \%$ acetonitrile, $0.1 \%$ formic acid for analysis. Peptides were then injected onto an Acclaim Pepmap $100 \mathrm{C} 18$ trap column $(2 \mathrm{~cm} \times 100 \mu \mathrm{m}$, particle size $5 \mu \mathrm{m}$, Thermo Fischer Scientific $)$ using an Easy-nano-LC 1000 liquid chromatography system and separated with an acetonitrile gradient on a $75-\mu \mathrm{m}$ Reprosil-Pur C18-AQ column with a $300 \mathrm{nl} / \mathrm{min}$ 
flow rate. Fractions were analyzed on Orbitrap Fusion Tribrid mass spectrometer (Thermo Fisher Scientific). Precursor ion mass spectra were acquired at 120.000 resolution and mass spectrometry (MS)/MS analysis was performed in a datadependent multinotch mode where collision-induced dissociation spectra of the most intense precursor ions were recorded in ion trap at collision energy setting of 30 for $3 \mathrm{~s}$. Charge states 2-7 were selected for fragmentation, dynamic exclusion was set to $30 \mathrm{~s}$. MS ${ }^{3}$ spectra for reporter ion quantitation were recorded at 60,000 resolution with higher energy collisional dissociation fragmentation at collision energy of 55 using synchronous precursor selection. In a second nano-LCMS analysis a list containing theoretical peptides from Iqgap1 and Tiam1 were included in the analysis.

The data files for the set were merged for identification and relative quantification using Proteome Discoverer version 1.4 (Thermo Fisher Scientific). The search was against the Mus musculus Swissprot Database version November 2017 (Swiss Institute of Bioinformatics, Switzerland) using Mascot 2.5 (Matrix Science) as a search engine with precursor mass tolerance of $5 \mathrm{ppm}$ and fragment mass tolerance of $0.5 \mathrm{Da}$. Tryptic peptides were accepted with zero missed cleavage and variable modifications of methionine oxidation, fixed cysteine alkylation, and TMT-label modifications of N-termini and lysines were selected. The sum of the control samples was used as denominator and for calculating ratios. The detected peptide threshold in the software was set to a minimum quantification threshold value of 1000 and a $1 \%$ false discovery rate by searching against a reversed database and grouping identified proteins by shared sequences to minimize redundancy. Only peptides unique for a given protein were considered for identification; peptides common to other isoforms or proteins of the same family were excluded.

F-actin staining. Macrophages were fixed with ice-cold methanol in chamber slides and stained with Alexa Fluor 488 Phalloidin (A12379, Thermo Fischer Scientific) for $30 \mathrm{~min}$. The slides were mounted with Prolong Gold Antifade Mounting reagent with DAPI (P36935, Thermo Fischer Scientific) and analyzed with confocal microscopy (LSM700, Zeiss).

Generating RhoGDI1-knockout macrophages. Recombinant CAS9 was purified from BL21 Escherichia coli (C600003, ThermoFisher Scientific) transduced with a plasmid encoding Streptococcus pyogenes CAS9 (gift from Niels Geijsen, Addgene plasmid \#62731). Synthesized crRNA:tracrRNA (Integrated DNA Technologies) targeting mouse Arghdia exon 2 (sg1: CAGAUAGCUGCAGAGAAUG, sg2: CUGCGCAAGCUGCUCAGCAG; retrieved from benchling.com) were preincubated with CAS9 in PBS for 10 min to create readily transfectable ribonucleic proteins (RNP). Immortalized macrophages ${ }^{45}$ were transfected with the RNPs using Lipofectamine RNAiMAX (13778150, ThermoFisher Scientific) during $48 \mathrm{~h}$, and pools of cells were used for analyses.

Generating Rac1-CAAX mutants with CRISPR/CAS9 editing. We used a single plasmid approach to edit the CAAX sequence of Racl. A CRISPR (cr) RNA template oligonucleotide 5'-GCTGAGACATTTACAACAGC-3' and its complementary fragment targeting exon 6 of Racl were annealed and cloned into the GeneArt-CRISPR Nuclease vector (Life Technology) downstream of a human U6 promoter. A 1-kb DNA fragment for homologous recombination-based editing was synthesized and cloned into unique $\mathrm{MfeI}$ and SpeI restriction sites of the vector; the fragment was composed of a 15-18-bp sequence encoding the mutant CAAX-motif (i.e., $5^{\prime}$-CGAAAGAGAAAATCTTTA-3' for $C-L-L-L$ to $S-L-L-L$ editing, and $5^{\prime}$-CGAAAGAGAAAATGC- $3^{\prime}$ for $C-L-L-L$ to $C-L-S T O P$ editing) and $0.5 \mathrm{~kb}$ flanking genomic sequences. In the resulting all-in-one gRNA-CAS9-editing plasmid the U6 promoter drives gRNA transcription; a cytomegalovirus promoter drives bicistronic expression of CAS9 and orange fluorescent protein linked by the 2A self-cleaving peptide; and the donor DNA sequence can replace the endogenous sequence by homologous recombination after CAS9 cleavage.

The vector was transiently transfected with jetPRIME (Polyplus) into HEK cells (HEK293, ATCC CRL-1573), and transfected single cells were sorted by fluorescence-activated cell sorting and plated in individual wells of 96-well plates. After 2-4 weeks, clones were expanded for cryopreservation and genomic DNA extraction (DNeasy blood \& tissue kit, Qiagen). Knock-in events were first detected by mutation-specific PCR with forward primer $5^{\prime}$-CTGTCCCAACACTCCCA TCAT- $3^{\prime}$ (binding genomic DNA upstream of the $5^{\prime}$-homology arm) and reverse primers 5'-AACAGTAAAGATTTTCTCTTTCG-3' (to detect the -SLLL editing) or 5'-TTACAAGCATTTTCTCTTTCG-3' (to detect -CL editing). Second, another PCR product from potential targets-amplified with forward oligo $5^{\prime}$-GTGGTCGT GTTTCCTGTAGGT-3' and reverse oligo 5'-AGTTCAGTGCTCGGTGTTCTC-3' -was TA-cloned and the plasmid of 10-12 transformed bacterial colonies for each potential target cell line was sequenced. And third, total RNA was isolated from the cells and a cDNA fragment was amplified with forward oligo 5'-CAAGTGTGTG GTGGTGGGAGA-3' and reverse oligo 5'-AACGAGGGGCTGAGACATTTA-3', and then sequenced using the forward oligo. Subsequently, three CAAX mutant (CM) cell lines were selected for experiments: RaclCM1, -2 , and -3 . Based on the DNA and cDNA sequencing, Rac1CM1 was predicted to have a Rac1 ${ }^{\text {SLLL/CLL }}$ genotype; $C M 2, R a c 1^{\mathrm{CL} / \mathrm{CL}}$; and $C M 3, R a 1^{\mathrm{CL} /-}$. A Rac1Ctr clone, was used as a manipulated $\mathrm{Racl}^{+/+}$control.
Statistics. Values are mean and SEM unless stated otherwise. Differences between groups were determined with Student's $t$ test or one-way ANOVA with Tukey's post hoc test, and were considered significant when $P<0.05$.

Reporting summary. Further information on research design is available in the Nature Research Reporting Summary linked to this article.

\section{Data availability}

The authors declare that the data supporting the findings of this study are available within the Article, supplementary information files and Source data, or are available upon reasonable requests to the authors.

Received: 4 December 2018 Accepted: 23 July 2019

Published online: 04 September 2019

\section{References}

1. Zhang, F. L. \& Casey, P. J. Influence of metal ions on substrate binding and catalytic activity of mammalian protein geranylgeranyltransferase type-I. Biochem. J. 320(Pt 3), 925-932 (1996).

2. Hori, Y. et al. Post-translational modifications of the C-terminal region of the rho protein are important for its interaction with membranes and the stimulatory and inhibitory GDP/GTP exchange proteins. Oncogene 6, 515-522 (1991).

3. Solski, P. A., Helms, W., Keely, P. J., Su, L. \& Der, C. J. RhoA biological activity is dependent on prenylation but independent of specific isoprenoid modification. Cell Growth Differ. 13, 363-373 (2002).

4. Sahai, E. \& Marshall, C. J. Rho-GTPases and cancer. Nat. Rev. Cancer 2, 133-142 (2002).

5. Kusama, T. et al. Selective inhibition of cancer cell invasion by a geranylgeranyltransferase-I inhibitor. Clin. Exp. Metastasis 20, 561-567 (2003).

6. Sjogren, A. K. et al. GGTase-I deficiency reduces tumor formation and improves survival in mice with K-Ras-induced lung cancer. J. Clin. Investig. 117, 1294-1304 (2007).

7. Heasman, S. J. \& Ridley, A. J. Mammalian Rho GTPases: new insights into their functions from in vivo studies. Nat. Rev. Mol. Cell Biol. 9, 690-701 (2008).

8. Connor, A. M., Berger, S., Narendran, A. \& Keystone, E. C. Inhibition of protein geranylgeranylation induces apoptosis in synovial fibroblasts. Arthritis Res. Ther. 8, R94 (2006).

9. Nagashima, T., Okazaki, H., Yudoh, K., Matsuno, H. \& Minota, S. Apoptosis of rheumatoid synovial cells by statins through the blocking of protein geranylgeranylation: a potential therapeutic approach to rheumatoid arthritis. Arthritis Rheum. 54, 579-586 (2006).

10. Walters, C. E. et al. Inhibition of Rho GTPases with protein prenyltransferase inhibitors prevents leukocyte recruitment to the central nervous system and attenuates clinical signs of disease in an animal model of multiple sclerosis. $J$. Immunol. 168, 4087-4094 (2002).

11. Jain, M. K. \& Ridker, P. M. Anti-inflammatory effects of statins: clinical evidence and basic mechanisms. Nat. Rev. Drug Discov. 4, 977-987 (2005).

12. Greenwood, J., Steinman, L. \& Zamvil, S. S. Statin therapy and autoimmune disease: from protein prenylation to immunomodulation. Nat. Rev. Immunol. 6, 358-370 (2006)

13. Winter-Vann, A. M. \& Casey, P. J. Post-prenylation-processing enzymes as new targets in oncogenesis. Nat. Rev. Cancer 5, 405-412 (2005).

14. Khan, O. M. et al. Geranylgeranyltransferase type I (GGTase-I) deficiency hyperactivates macrophages and induces erosive arthritis in mice. J. Clin. Investig. 121, 628-639 (2011).

15. Khan, O. M. et al. Targeting GGTase-I activates RhoA, increases macrophage reverse cholesterol transport, and reduces atherosclerosis in mice. Circulation 127, 782-790 (2013).

16. Philips, M. R. The perplexing case of the geranylgeranyl transferase-deficient mouse. J. Clin. Investig. 121, 510-513 (2011).

17. Taylor, J. S., Reid, T. S., Terry, K. L., Casey, P. J. \& Beese, L. S. Structure of mammalian protein geranylgeranyltransferase type-I. Embo J. 22, 5963-5974 (2003).

18. Bos, J. L., Rehmann, H. \& Wittinghofer, A. GEFs and GAPs: critical elements in the control of small G proteins. Cell 129, 865-877 (2007).

19. Briggs, M. W. \& Sacks, D. B. IQGAP proteins are integral components of cytoskeletal regulation. EMBO Rep. 4, 571-574 (2003).

20. Brown, M. D. \& Sacks, D. B. Iqgap1 in cellular signaling: bridging the GAP. Trends Cell Biol. 16, 242-249 (2006).

21. Garcia-Mata, R., Boulter, E. \& Burridge, K. The 'invisible hand': regulation of Rho GTPases by RhoGDIs. Nat. Rev. Mol. Cell Biol. 12, 493-504 (2011).

22. Boulter, E. et al. Regulation of Rho GTPase crosstalk, degradation and activity by RhoGDI1. Nat. Cell Biol. 12, 477-483 (2010). 
23. Kuijk, L. M. et al. HMG-CoA reductase inhibition induces Il-1beta release through Rac1/PI3K/PKB-dependent caspase-1 activation. Blood 112, 3563-3573 (2008).

24. Ghiaur, G. et al. Racl is essential for intraembryonic hematopoiesis and for the initial seeding of fetal liver with definitive hematopoietic progenitor cells. Blood 111, 3313-3321 (2008).

25. Wells, C. M., Walmsley, M., Ooi, S., Tybulewicz, V. \& Ridley, A. J. Rac1deficient macrophages exhibit defects in cell spreading and membrane ruffling but not migration. J. Cell Sci. 117, 1259-1268 (2004).

26. Tan, W. et al. An essential role for Racl in endothelial cell function and vascular development. FASEB J. 22, 1829-1838 (2008).

27. Satoh, M. et al. Requirement of Rac1 in the development of cardiac hypertrophy. Proc. Natl Acad. Sci. USA 103, 7432-7437 (2006).

28. Wang, G. et al. Genetic ablation of Rac1 in cartilage results in chondrodysplasia. Dev. Biol. 306, 612-623 (2007).

29. Abu-Issa, R. Racl modulates cardiomyocyte adhesion during mouse embryonic development. Biochem. Biophys. Res. Commun. 456, 847-852 (2015).

30. Wisniewski, J. R., Zougman, A., Nagaraj, N. \& Mann, M. Universal sample preparation method for proteome analysis. Nat. Methods 6, 359-362 (2009).

31. Bashour, A. M., Fullerton, A. T., Hart, M. J. \& Bloom, G. S. Iqgap1, a Rac- and Cdc42-binding protein, directly binds and cross-links microfilaments. J. Cell Biol. 137, 1555-1566 (1997).

32. Casteel, D. E. et al. Rho isoform-specific interaction with Iqgap1 promotes breast cancer cell proliferation and migration. J. Biol. Chem. 287, 38367-38378 (2012).

33. Li, S., Wang, Q., Chakladar, A., Bronson, R. T. \& Bernards, A. Gastric hyperplasia in mice lacking the putative Cdc42 effector Iqgap1. Mol. Cell. Biol. 20, 697-701 (2000).

34. Wang, M. \& Casey, P. J. Protein prenylation: unique fats make their mark on biology. Nat. Rev. Mol. Cell Biol. 17, 110-122 (2016).

35. Lopez-Castejon, G. \& Brough, D. Understanding the mechanism of Il-1beta secretion. Cytokine Growth Factor Rev. 22, 189-195 (2011).

36. Cullen, S. P., Kearney, C. J., Clancy, D. M. \& Martin, S. J. Diverse activators of the NLRP3 inflammasome promote Il-1beta secretion by triggering necrosis. Cell Rep. 11, 1535-1548 (2015).

37. Yoon, S. et al. Stat 3 transcriptional factor activated by reactive oxygen species induces Il6 in starvation-induced autophagy of cancer cells. Autophagy 6 , $1125-1138$ (2010).

38. Liu, J. et al. The ROS-mediated activation of Il-6/Stat3 signaling pathway is involved in the 27-hydroxycholesterol-induced cellular senescence in nerve cells. Toxicol. Vitr. 45, 10-18 (2017).

39. Simon, A. R. et al. Regulation of Stat3 by direct binding to the Rac1 GTPase. Science 290, 144-147 (2000).

40. Oike, T. et al. Stat 3 as a potential therapeutic target for rheumatoid arthritis. Sci. Rep. 7, 10965 (2017).

41. Ogura, $\mathrm{H}$. et al. Interleukin-17 promotes autoimmunity by triggering a positive-feedback loop via interleukin-6 induction. Immunity 29, 628-636 (2008).

42. Yang, Y. et al. Functional roles of p38 mitogen-activated protein kinase in macrophage-mediated inflammatory responses. Mediat. Inflamm. 2014 352371 (2014)

43. Irtegun, S. et al. Pharmacological inactivation of Src family kinases inhibits LPS-induced Tnf-alpha production in PBMC of patients with Behcet's disease. Mediat. Inflamm. 2016, 5414369 (2016).

44. Byeon, S. E. et al. The role of Src kinase in macrophage-mediated inflammatory responses. Mediat. Inflamm. 2012, 512926 (2012).

45. Akula, M. K. et al. Control of the innate immune response by the mevalonate pathway. Nat. Immunol. 17, 922-929 (2016).

46. Henneman, L., Schneiders, M. S., Turkenburg, M. \& Waterham, H. R. Compromized geranylgeranylation of RhoA and Racl in mevalonate kinase deficiency. J. Inherit. Metab. Dis. 33, 625-632 (2010).

47. Munoz, M. A. et al. Defective protein prenylation is a diagnostic biomarker of mevalonate kinase deficiency. J. Allergy Clin. Immunol. 140, 873-875 e876 (2017).

48. Lindholm, M. W. \& Nilsson, J. Simvastatin stimulates macrophage interleukin-1beta secretion through an isoprenylation-dependent mechanism. Vascul. Pharmacol. 46, 91-96 (2007).

49. Bjorkhem-Bergman, L., Lindh, J. D. \& Bergman, P. What is a relevant statin concentration in cell experiments claiming pleiotropic effects? Br. J. Clin. Pharm. 72, 164-165 (2011)

50. Youssef, S. et al. The HMG-CoA reductase inhibitor, atorvastatin, promotes a Th2 bias and reverses paralysis in central nervous system autoimmune disease. Nature 420, 78-84 (2002).

51. Chow, O. A. et al. Statins enhance formation of phagocyte extracellular traps. Cell Host Microbe 8, 445-454 (2010).

52. Metais, C., Hughes, B. \& Herron, C. E. Simvastatin increases excitability in the hippocampus via a PI3 kinase-dependent mechanism. Neuroscience 291, 279-288 (2015).

53. Chrostek, A. et al. Racl is crucial for hair follicle integrity but is not essential for maintenance of the epidermis. Mol. Cell Biol. 26, 6957-6970 (2006).
54. Jackson, B. et al. RhoA is dispensable for skin development, but crucial for contraction and directed migration of keratinocytes. Mol. Biol. Cell 22, 593-605 (2011)

55. $\mathrm{Wu}, \mathrm{X}$. et al. Cdc42 controls progenitor cell differentiation and beta-catenin turnover in skin. Genes Dev. 20, 571-585 (2006).

56. Andersson, K. M., Svensson, M. N., Erlandsson, M. C., Jonsson, I. M. \& Bokarewa, M. I. Down-regulation of survivin alleviates experimental arthritis. J. Leukoc. Biol. 97, 135-145 (2015).

57. Takeshita, S., Kaji, K. \& Kudo, A. Identification and characterization of the new osteoclast progenitor with macrophage phenotypes being able to differentiate into mature osteoclasts. J. Bone Min. Res. 15, 1477-1488 (2000).

58. Taguchi, Y. \& Schatzl, H. M. Small-scale triton X-114 extraction of hydrophobic proteins. Bio Protoc. 4, el139 (2014).

59. Long, D. L., Willey, J. S. \& Loeser, R. F. Racl is required for matrix metalloproteinase 13 production by chondrocytes in response to fibronectin fragments. Arthritis Rheum. 65, 1561-1568 (2013).

60. Nishimura, A. \& Linder, M. E. Identification of a novel prenyl and palmitoyl modification at the CaaX motif of Cdc42 that regulates RhoGDI binding. Mol Cell. Biol. 33, 1417-1429 (2013).

61. Choo, Y. S. \& Zhang, Z. Detection of protein ubiquitination. J. Vis. Exp. 19 1293 (2009).

\section{Acknowledgements}

This study was supported by grants from the Knut and Alice Wallenberg Foundation, Strategic Research Program in Cancer at Karolinska Institutet, Center for Innovative Medicine (CIMED), Swedish Cancer Society, and Swedish Heart \& Lung Foundation (to M.O.B.); the Swedish Research Council (to M.O.B, V.I.S., and M.B.); the Swedish Society for Medical Research, Knut and Alice Wallenberg Foundation, and Wallenberg Centre for Molecular and Translational Medicine (to V.I.S); and the Swedish Rheumatism Association (to M.B.). The Proteomics Core Facility at the Sahlgrenska Academy, Gothenburg University, performed the mass spectrometry analyses. Open access funding provided by University of Gothenburg.

\section{Author contributions}

M.K.A. designed the study, performed experiments, interpreted data, made figures and statistics, and wrote the first draft of the manuscript; M.X.I. designed and performed experiments, and prepared figures; E.G.I. designed and performed the experiments, and prepared the figures; O.M.K. designed and performed the experiments; I.T.K. performed the experiments; M.E. performed the histology; C.K. performed the histology and provided technical assistance; X.X. performed the CRISPR/CAS9 gene editing; M.Br. designed the experiments; C.B. provided the mouse models; M.Bo. designed the experiments and quantified arthritis; D.W. designed the experiments and provided the reagents; V.I.S. interpreted data and provided funding; and M.O.B. designed and supervised the study, provided funding, and wrote the paper.

\section{Additional information}

Supplementary Information accompanies this paper at https://doi.org/10.1038/s41467 019-11606-x.

Competing interests: The authors declare no competing interests.

Reprints and permission information is available online at http://npg.nature.com/ reprintsandpermissions/

Peer review information: Nature Communications thanks Carol Williams and other anonymous reviewer(s) for their contribution to the peer review of this work. Peer reviewer reports are available.

Publisher's note: Springer Nature remains neutral with regard to jurisdictional claims in published maps and institutional affiliations.

Open Access This article is licensed under a Creative Commons Attribution 4.0 International License, which permits use, sharing, adaptation, distribution and reproduction in any medium or format, as long as you give appropriate credit to the original author(s) and the source, provide a link to the Creative Commons license, and indicate if changes were made. The images or other third party material in this article are included in the article's Creative Commons license, unless indicated otherwise in a credit line to the material. If material is not included in the article's Creative Commons license and your intended use is not permitted by statutory regulation or exceeds the permitted use, you will need to obtain permission directly from the copyright holder. To view a copy of this license, visit http://creativecommons.org/ licenses/by/4.0/

(C) The Author(s) 2019 